\title{
RESOLVING THE MULTITUDE OF MICROSCALE INTERACTIONS ACCURATELY MODELS STOCHASTIC PARTIAL DIFFERENTIAL EQUATIONS
}

\section{A. J. ROBERTS}

\begin{abstract}
Constructing numerical models of noisy partial differential equations is a very delicate task. Our long-term aim is to use modern dynamical systems theory to derive discretisations of dissipative stochastic partial differential equations. As a second step, we consider here a small domain, representing a finite element, and derive a one-degreeof-freedom model for the dynamics in the element; stochastic centre manifold theory supports the model. The approach automatically parametrises the microscale structures induced by spatially varying stochastic noise within the element. The crucial aspect of this work is that we explore how a multitude of microscale noise processes may interact in nonlinear dynamical systems. The analysis finds that noise processes with coarse structure across a finite element are the significant noises for the modelling. Further, the nonlinear dynamics abstracts effectively new noise sources over the macroscale timescales resolved by the model.
\end{abstract}

\section{Introduction}

We need, accurately and efficiently, to model numerically the evolution of stochastic partial differential equations (SPDEs). For example, SPDEs may be used to model pattern-forming systems $[\mathbf{1 5}, \mathbf{6}]$. Due to the forcing over many length- and timescales, an SPDE typically has intricate spatio-temporal dynamics. Numerical methods to integrate stochastic ordinary differential equations are known to be delicate and subtle (see, for example, [21]). We surely must take considerable care for SPDEs as well (see, for example, $[\mathbf{1 8}, \mathbf{4 2}]$ ).

A critical aspect of SPDEs is that the stochastic effects generate high-wavenumber, steep variations, in spatial structures. However, stable time integration schemes generally damp such high-wavenumber decaying modes far too fast. Thus stable time integration schemes often predict these high-wavenumber modes to be far too small. Nevertheless, through stochastic resonance an accurate resolution of the amplitude and life-time of these highwavenumber modes may be important on the large-scale dynamics. The term 'stochastic resonance' includes phenomena where stochastic fluctuations interact with each other and themselves through nonlinearity in the dynamical system, not only to generate long timedrifts but also to change stability (see, for example, $[22,7, \mathbf{1 5}, \mathbf{3 5}, \mathbf{4 1}]$ ) as seen in the model SDE (4). Thus, for accurate modelling of SPDEs, we must reasonably resolve the high-wavenumber microscale structures. Only then will numerical discretisation on large 
space-time grids achieve efficiency without sacrificing the subtle interactions that take place among stochastic subgrid scale structures.

Centre manifold theory supports the macroscale modelling of microscale dynamics. For example, Knobloch \& Wiesenfeld [22], Boxler [7, 8] and Arnold [1, §7.6] explicitly used stochastic centre manifold (SCM) theory to support the modelling of SDEs. Indeed, Boxler [7, p. 510] proves that 'stochastic center manifolds share all the nice properties of their deterministic counterparts'. Others, such as Berglund \& Gentz [5], Blömker, Hairer \& Pavliotis [6], and Kabanov \& Pergamenshchikov [20], use the same separation of timescales that underlies centre manifold theory to form and support low-dimensional, long-term models of SDES and SPDEs that have both fast and slow modes. A centre manifold approach also illuminates the discretisation of deterministic partial differential equations $[30,32,23,31,33,24]$. By merging these two applications of centre manifold theory, we may model SPDEs with sound theoretical support. Here we begin to explore the discretisation of SPDEs based upon SCM theory. We consider the case of just one finite element forming the domain. Later work will address how to couple many finite elements together to form large-scale discrete models of SPDES.

The crucial issue explored here is how to deal with noise that is distributed independently across space as well as time; that is, the noise is uncorrelated in space and time. We decompose the noise into its Fourier sine series, and assume that the infinite number of Fourier coefficients are an infinite number of independent noise sources. The word 'multitude' in the title reflects the vast number of nonlinear interactions among the infinite number of these Fourier modes. It eventuates that only a finite number of combinations of these noise sources are significant in the model. However, all do contribute in the infinite sums forming these few combinations.

The importance of this work is to establish a practical methodology to create accurate, finite-dimensional, discrete models of the long-term dynamics of SPDEs.

\subsection{Analyse a prototype SPDE}

Continuing earlier work [35], we explore the modelling of the dynamics of a SPDE on just one finite-size element — the simplest case. As a prototype application of the methodology, let us consider the stochastically forced nonlinear partial differential equation

$$
\frac{\partial u}{\partial t}=-u \frac{\partial u}{\partial x}+\frac{\partial^{2} u}{\partial x^{2}}+u+\sigma \phi(x, t), \quad \text { such that } u=0 \text { at } x=0, \pi,
$$

which involves the important generic physical processes of nonlinear advection $u u_{x}$, linear diffusion $u_{x x}$, some additive noise process $\phi(x, t)$, and a linear reaction $u$. The reaction term partially ameliorates diffusion to make the $\sin x$ mode dynamically neutral. Analogously, Blömker et al. [6] rigorously modelled the stochastically forced Swift-Hohenberg equation by a stochastic Ginzburg-Landau equation as a prototype SPDE in a class of pattern-forming stochastic systems. Consider the forcing $\phi(x, t)$, of strength $\sigma$, to be a white-noise process that is delta-correlated in both space and time; although note that Sections 2 and 3 actually apply to much more generic forcing. Express the additive noise in the orthogonal sine series

$$
\phi(x, t)=\sum_{k=1}^{\infty} \phi_{k}(t) \sin k x
$$

where the $\phi_{k}(t)$ are independent white noises that are delta-correlated in time. (The reason for expressing the noise in the sine expansion (2) is that the modes $\sin k x$ are the eigenmodes 
of the linear dynamics and thus form a natural basis for analysis.) These spatially distributed noises interact through the nonlinearity of the prototype SPDE (1). Our immediate aim is to discover how these noise interactions affect the dynamics over the relatively large-scale domain $[0, \pi]$.

Throughout the body of this paper we interpret all noise processes and all stochastic differential equations in the Stratonovich sense so that the rules of traditional calculus apply. Thus the direct application of this modelling is to physical systems where the Stratonovich interpretation is the norm. However, Appendix B provides alternative derivations of some key properties of the nonlinear interaction of noise processes: these derivations use the Ito interpretation. Only in Appendix B is the Ito interpretation used; everywhere else the stochastic calculus is Stratonovich.

\subsection{Centre manifold theory supports modelling}

We base the macroscale modelling upon the dynamics when the noise is absent, $\sigma=0$. When $\sigma=0$, the linear dynamics of the SPDE (1), namely

$$
\frac{\partial u}{\partial t}=\frac{\partial^{2} u}{\partial x^{2}}+u \quad \text { such that } u=0 \text { at } x=0, \pi,
$$

are that modes $u \propto \sin k x \exp \lambda t$ decay with rate $\lambda_{k}=-\left(k^{2}-1\right)$ except for the $k=1$ mode, $u \propto \sin x$, which is linearly neutral, $\lambda_{1}=0$, and thus forms the basis of the long-term model. Those components of the forcing noise (2) with wavenumber $k>1$ are orthogonal to this basic mode. Consequently, simple numerical methods, such as Galerkin projection onto the fundamental mode $\sin x$, would ignore the 'high-wavenumber' modes, $k>1$, of the noise (2) and hence would completely miss subtle but important subgrid interactions. Instead, the systematic nature of SCM theory accounts for the subgrid-scale interactions as a series in the noise amplitude $\sigma$ from the deterministic base (3).

SCM theory, summarised in Appendix A, applies to the nonlinear, stochastically forced SPDE (1) because in the linearised PDE (3) there is some (here, one) eigenvalue of 0 and all the other eigenvalues are negative (and bounded away from 0). After adjoining the trivial $d \sigma / d t=0, \mathrm{SCM}$ theory assures us (see Theorems 2 and 3 ) that in some finite neighbourhood of $(u, \sigma)=(0,0)$ there exists a slow manifold $u=v(a(t), x, t, \sigma)$ where the amplitude $a$ of the neutral mode $\sin x$ evolves according to a low-dimensional 'model' SDE $\dot{a}=g(a, t, \sigma)$ for some function $g$. Unfortunately, there is a caveat: Boxler's [7] and Arnold's [1, Chapter 7] SCM theory is as yet developed only for finite-dimensional systems which satisfy a Lipschitz condition. Here, the SPDE (1) is infinite-dimensional, and furthermore the nonlinear advection $u \partial u / \partial x$ involves the unbounded operator $\partial / \partial x$. There is some infinite-dimensional theory: Blömker et al. [6, Theorem 1.2] rigorously proved the existence and relevance of a stochastic Ginzburg-Landau model to the stochastic forced Swift-Hohenberg PDE; further, Caraballo, Langa \& Robinson [9] and Duan, Lu \& Schmalfuss [16] proved the existence of invariant manifolds for a wide class of reactiondiffusion SPDEs; they built on earlier work on inertial manifolds in SPDEs by Bensoussan \& Flandoli [4]. I expect future theoretical developments to rigorously support the modelling of SPDES.

However, in the interim, let us proceed via a shadowing argument. The high-wavenumber modes of the SPDE (1) dissipate rapidly; the spectrum $\lambda_{k} \sim-k^{2}$ for large-wavenumber $k$. This rapid dissipation ensures that the dynamics of the SPDE (1) is close to finite-dimensional. By modifying the spatial derivatives in the SPDE (1) to have a high-wavenumber cutoff, the 
dynamics of the cutoff version of (1) is effectively that of a Lipschitz, finite-dimensional, system. The SCM theorems of Boxler [7] then rigorously apply. For example, Section 4 shows that using just ten spatial modes in the noise $\phi(x, t)$ gives the coefficients in the stochastic model (24) correct to five decimal digits. Thus modifying $\partial / \partial x$ to cutoff modes with wavenumber $k>20$ forms a nearby, Lipschitz, finite-dimensional SDE system that is effectively indistinguishable from the original SPDE to five decimal digits, and to the order of asymptotic expansion pursued here. Whenever theoretical support is invoked, I actually refer to the nearby system of this projection of SPDE (1) onto a subspace of large but finite dimensions.

\subsection{Stochastic induced drift affects stability}

Previously [35], I pointed out that when the $\sin 2 x$ component $\phi_{2}(t)$ of the noise (2) is large enough, and in the absence of any other noise component $\left(\phi_{k}=0\right.$ for $\left.k \neq 2\right)$, then stochastic resonance may make a qualitative change in the nature of the solutions of the SPDE (1) in that stochastic resonance restabilises the zero equilibrium. The stochastic model described the evolution of the amplitude $a(t)$ of the $\sin x$ mode as the SDE

$$
\dot{a} \approx-\frac{\sigma^{2}}{88} a-\frac{1}{12} a^{3}+\frac{1}{6} \sigma a \phi_{2}+\frac{\sqrt{515}}{1936 \sqrt{3}} \sigma^{2} a \Phi,
$$

for some white noise $\Phi(t)$ independent of $\phi_{2}$ over long times. The second key theorem of SCMs is that models such as the SDE (4) do capture the long-term dynamics of the original stochastic SPDE (1). For example, Theorem 4 assures us that all nearby solutions of the SPDE (1) approach, exponentially quickly in time, a solution of the model SDE (4) embedded on the slow manifold $u=v(a(t), x, t, \sigma)$. This theorem assures us that apart from exponentially decaying transients, models such as the SDE (4) describe all the long-term dynamics of the full SPDE.

The nonlinearity of the SPDE acting on the stochastic forcing generates two new effects. First, it generates the effectively new multiplicative noise, $\propto \sigma^{2} a \Phi$. Second, and often its most significant effect, is the enhancement of the stability of the equilibrium $a=0$ through the $-\sigma^{2} a / 88$ term. In other examples, Boxler [7, p. 544], Drolet \& Vinals [14, 15], Knobloch \& Weisenfeld [22], and Vanden-Eijnden [41, p. 68] found the same sort of stability-modifying linear term in their analyses of stochastically perturbed bifurcations. Boxler [7, Theorem 7.3(a)] and Arnold [1, Corollary 7.4.7] prove that the stability of an SDE is the same as the model SDE on the SCM. Analogously, Just et al. [19] sought to determine how microtime deterministic chaos, instead of the noise that we consider here, translates into a new, effective stochastic noise in the slow modes of a deterministic dynamical system. Here, we explore the modelling of induced changes to the stability of the SPDE (1) through the transformation by nonlinearity of microscale noise into macroscale drift and noise. Indeed, our more complete analysis in Sections 3 and 4 shows that noises of wavenumbers $k \neq 2$ all contribute to destabilise the equilibrium; see the model SDE (24).

\subsection{The approach}

For the first part of the analysis of the SPDE (1), namely Sections 2 and 3, the requirement of white, delta-correlated noise is irrelevant; the results are valid for quite general timedependent, additive forcing. Section 2 shows how to remove 'memory' convolutions over the past history of terms linear in the noise. However, in a nonlinear system there are effects that are quadratic in the noise processes; transformations in Section 3 reduce the number 
of memory convolutions of quadratic noise terms, but cannot eliminate all such memory convolutions.

In the second part of the analysis (Sections 4 and 5), the critical assumption of white, delta-correlated noise enables simplifying analysis of the nonlinear interactions. We consider the Fokker-Planck equations for the probability density functions (PDFs) of the irreducible quadratic noise processes. Deterministic centre manifold modelling shows that a PDF approximately factors into a multivariate Gaussian quasi-stationary distribution (see, for example, [27]) and a slowly evolving conditional probability. Such a factorisation is also the key to the modelling by Just et al. [19] of fast deterministic chaos as noise on the slow modes. This factorisation effectively abstracts new noise processes over the long timescales of interest in the model. Section 4 discusses the specifics, such as an appropriate version of a model SDE, such as (4), for the SPDE (1) with delta-correlated noise in space and time, whereas Section 5 presents generic transformations of the irreducible quadratic noises for use in analysing general stochastic systems. Appendix B provides alternate derivations, using Ito calculus, of some of the key results on the modelling of nonlinear interactions among the noise components.

\section{Construction of a memoryless normal form model}

The linearised PDE (3) identifies that the long-term dynamics of the SPDE (1) may be parametrised by the amplitude $a(t)$ of the neutral mode $\sin x$. In this section, SCM techniques are used to construct the model. Arnold et al. [2] investigated stochastic Hopf bifurcations in this way, and the approach is equivalent to the slaving principle for SDEs used by Schoner $\&$ Haken [38]. However, most scientists generate models with convolutions over fast timescales of the noise. Here we simplify the model SDE tremendously by removing such 'memory' convolutions. This removal of convolutions was originally developed for SDEs by Coullet, Elphick \& Tirapegui [13], Sri Namachchivaya \& Lin [39], and Roberts \& Chao [10, 35].

SCM theory supports the modelling. Critical interesting features of the model reflect the steps taken to construct the model; thus the next two sections discuss the iterative construction of the SCM model. Note that the amplitude $a$ and the noise intensity $\sigma$ are the small parameters in the asymptotic expansions forming the model. Theorem 5 assures us that if we satisfy the SPDE (1) to some residual $\mathcal{O}\left(\|(a, \sigma)\|^{q}\right)$, then the stochastic slow manifold and the evolution thereon have the same order of error. The support that Theorem 5 gives to our modelling is independent of the details of construction. One complication is that I construct models to residuals of $\mathcal{O}\left(a^{4}+\sigma^{2}\right)$, for example. The theory covers this when we simply define a new small parameter $\varepsilon=\sqrt{\sigma}$; then, for example, a residual of $\mathcal{O}\left(a^{4}+\sigma^{2}\right)=\mathcal{O}\left(\|(a, \varepsilon)\|^{4}\right)$; hence Theorem 5 applies to assure us the errors in the SCM model are of $\mathcal{O}\left(\|(a, \varepsilon)\|^{4}\right)=\mathcal{O}\left(a^{4}+\sigma^{2}\right)$. I use this latter form to report the residuals and errors. Because the critical aspect of constructing the slow manifold model is simply the ultimate order of the residual of the SPDE (1), the specific details of the computation are not recorded here. Instead, computer algebra $[36, \S 1]$ performs all the details. Here I just report on critical steps in the method.

Consider the task of iteratively constructing an SCM model for the SPDE (1) using iteration [29]. We seek solutions in the form $u=v(a, x, t, \sigma)=a \sin x+\cdots$ such that the amplitude $a$ evolves according to some model SDE $\dot{a}=g(a, t, \sigma)$, such as the SDE (4). The steps in the construction proceed iteratively. Suppose that at some stage we have an asymptotic approximation to the SCM model; then the next iteration is to seek small corrections, denoted $v^{\prime}$ and $g^{\prime}$, to improve the asymptotic approximation. As the iterations proceed, the 
small corrections $v^{\prime}$ and $g^{\prime}$ get systematically smaller — that is, of higher order in the small parameters $a$ and $\sigma$ of the asymptotic expansion. As explained in [29]: substitute $u=v+v^{\prime}$ and $\dot{a}=g+g^{\prime}$ into the SPDE (1); linearise the problem for $v^{\prime}$ and $g^{\prime}$ by dropping products of small corrections; approximate terms in $v^{\prime}$ and $g^{\prime}$ by replacing their coefficients by the leading order approximation; and find that the corrections should satisfy

$$
\frac{\partial v^{\prime}}{\partial t}-\frac{\partial^{2} v^{\prime}}{\partial x^{2}}-v^{\prime}+g^{\prime} \sin x=\operatorname{residual}_{(1)} .
$$

Here, the 'residual' is the residual of the SPDE (1), evaluated for the currently known asymptotic approximation. For example, if at some stage we had determined that the deterministic part of the SCM model was

$$
u=a \sin x-\frac{1}{6} a^{2} \sin 2 x+\frac{1}{32} a^{3} \sin 3 x+\mathcal{O}\left(a^{4}, \sigma\right)
$$

$$
\text { such that } \quad \dot{a}=-\frac{1}{12} a^{3}+\mathcal{O}\left(a^{4}, \sigma\right),
$$

then the residual of the SPDE (1) for the next iteration would be simply the stochastic forcing:

$$
\operatorname{residual}_{(1)}=\sigma \sum_{k=1}^{\infty} \phi_{k} \sin k x+\mathcal{O}\left(a^{4}\right) .
$$

At any iteration, the terms in the residual split into two categories, as follows, as is standard in singular perturbations.

- The components in $\sin k x$ for wavenumbers $k \geqslant 2$ cause no great difficulties. We include a corresponding component in the correction $v^{\prime}$ to the field in proportion to $\sin k x$. When the coefficient of $\sin k x$ in the residual is time-dependent, the component in the correction $v^{\prime}$ is $\mathscr{H}_{k} \phi_{k}(t) \sin k x$, in which the operator $\mathscr{H}_{k}$ denotes convolution over past history with $\exp \left[-\left(k^{2}-1\right) t\right]$; namely, $\mathscr{H}_{k} \phi=\exp \left[-\left(k^{2}-1\right) t\right] \star \phi(t)=$ $\int_{-\infty}^{t} \exp \left[-\left(k^{2}-1\right)(t-\tau)\right] \phi(\tau) d \tau$.

- However, any component in $\sin x$, such as $\phi_{1}$ in this iteration with this residual, must cause a contribution to the evolution correction $g^{\prime}$, here simply $g^{\prime}=\sigma \phi_{1}$, as no uniformly bounded component in $v^{\prime}$ of $\sin x$ can match a $\sin x$ component of the residual. This is the standard solvability condition for singular perturbations.

However, a more delicate issue arises in subsequent corrections. In the next iteration, the next residual is

$$
\begin{aligned}
\operatorname{residual}_{(1)}=a \sigma\left[\frac{1}{2} \mathscr{H}_{2} \phi_{2} \sin x\right. & +\left(\frac{1}{3} \phi_{1}+\mathscr{H}_{3} \phi_{3}\right) \sin 2 x \\
& \left.+\sum_{k=3}^{\infty} \frac{k}{2}\left(\mathscr{H}_{k+1} \phi_{k+1}-\mathscr{H}_{k-1} \phi_{k-1}\right) \sin k x\right]+\mathcal{O}\left(a^{4}+\sigma^{2}\right) .
\end{aligned}
$$

Many are tempted to simply use the solvability condition and match the $\sin x$ component in this residual directly by making the correction $a \sigma \frac{1}{2} \mathscr{H}_{2} \phi_{2}$ to the evolution $g^{\prime}$. But this choice introduces incongruous short-timescale convolutions of the forcing into the model SDE (4) of the long-time evolution. The appropriate alternative $[13, \mathbf{3 9}, \mathbf{1 0}, \mathbf{3 5}]$ is to recognise that part of the convolution can be integrated: since for any $\Phi(t)$ see that 
$d / d t \mathscr{H}_{k} \Phi=-\left(k^{2}-1\right) \mathscr{H}_{k} \Phi+\Phi ;$ thus

$$
\mathscr{H}_{k} \Phi=\frac{1}{k^{2}-1}\left[-\frac{d}{d t} \mathscr{H}_{k} \Phi+\Phi\right] .
$$

Consequently, we split such a convolution in the residual, when multiplied by the neutral mode $\sin x$, into:

- the first part of (6), namely $-d / d t \mathscr{H}_{k} \Phi /\left(k^{2}-1\right)$, to be integrated into the next update $v^{\prime}$ for the subgrid field; and

- the second part of (6), namely $\Phi /\left(k^{2}-1\right)$, to update the evolution via $g^{\prime}$.

For the example residual (5), the term $\frac{1}{2} a \sigma \mathscr{H}_{2} \phi_{2} \sin x$ in the residual thus forces a term $-\frac{1}{6} a \sigma \mathscr{H}_{2} \phi_{2} \sin x$ into the subgrid field, and a term $\frac{1}{6} a \sigma \phi_{2}$ into the model SDE for $\dot{a}$. When a component in the residual has many convolutions, then we apply this separation recursively. Such separation works whether the forcing is deterministic or stochastic. This separation removes all fast-time memory convolutions linear in the forcing from the evolution equation for the amplitude $a(t)$.

Continuing this iterative construction gives models to high order in the asymptotic expansion in the amplitude $a$. The iteration terminates when the residuals are zero to some specified order. Then the SCM Approximation Theorem 5 assures us that the model has the same order of error as the residual.

For example, terminating the iterative construction so that residual $(1)=\mathcal{O}\left(a^{4}+\sigma^{2}\right)$, we find that the stochastic slow manifold is

$$
\begin{aligned}
& u=a \sin x-\frac{1}{6} a^{2} \sin 2 x+ \frac{1}{32} a^{3} \sin 3 x+\sigma \sum_{k=2}^{\infty} \mathscr{H}_{k} \phi_{k} \sin k x \\
&+a \sigma[-\frac{1}{6} \mathscr{H}_{2} \phi_{2} \sin x+\left(\frac{1}{3} \mathscr{H}_{2} \phi_{1}+\mathscr{H}_{2} \mathscr{H}_{3} \phi_{3}\right) \sin 2 x \\
&\left.+\sum_{k=3}^{\infty} \frac{k}{2} \mathscr{H}_{k}\left(\mathscr{H}_{k+1} \phi_{k+1}-\mathscr{H}_{k-1} \phi_{k-1}\right) \sin k x\right] \\
&+\mathcal{O}\left(a^{4}+\sigma^{2}\right) .
\end{aligned}
$$

The corresponding model SDE for the evolution,

$$
\dot{a}=-\frac{1}{12} a^{3}+\sigma\left[\phi_{1}+\frac{1}{6} a \phi_{2}+a^{2}\left(\frac{1}{18} \phi_{1}+\frac{1}{96} \phi_{3}\right)\right]+\mathcal{O}\left(a^{5}+\sigma^{2}\right),
$$

has no fast-time convolutions, only the direct influence of the forcing. This is the preferred normal form for a noisy model.

Note the generic feature that the originally additive noise, through the nonlinearities in the system, appears as a multiplicative noise in the model. However, it is only the coarse structure of the forcing/noise that appears in the model: all components of the forcing/noise with wavenumber $k>3$ are ineffective in these, the most important, terms in a model.

\section{Irreducibility of quadratic interactions}

Consider continuing the iterative construction of the stochastic slow manifold model to determine effects that are quadratic in the magnitude $\sigma$ of the noise. We seek terms in $\sigma^{2}$, as these generate mean drift terms, and we also seek terms in $a \sigma^{2}$, as these affect the linear stability of the SPDE (1); see [35, Figure 2] and [7, p. 544]. 
Driven by the residuals of the SPDE (1), computer algebra [36, $\S \S 1.1-4]$ determines the model SDE to be

$$
\begin{aligned}
& \dot{a}=-\frac{1}{12} a^{3}-\frac{7}{3456} a^{5} \\
&+ \sigma \phi_{1}+\frac{1}{6} a \sigma \phi_{2}+a^{2} \sigma\left(\frac{1}{18} \phi_{1}+\frac{1}{96} \phi_{3}\right)+a^{3} \sigma\left(\frac{1}{54} \phi_{2}+\frac{1}{4320} \phi_{4}\right) \\
&+ \sigma^{2}\left[\frac{1}{6} \phi_{1} \mathscr{H}_{2} \phi_{2}+\sum_{k=2}^{\infty} \frac{\phi_{k} \mathscr{H}_{k+1} \phi_{k+1}+\phi_{k+1} \mathscr{H}_{k} \phi_{k}}{2\left(2 k^{2}+2 k-1\right)}\right] \\
&+a \sigma^{2}\left[\frac{1}{18} \phi_{1} \mathscr{H}_{2} \phi_{1}+\frac{19}{528} \phi_{1} \mathscr{H}_{3} \phi_{3}+\frac{1}{6} \phi_{1} \mathscr{H}_{2} \mathscr{H}_{3} \phi_{3}\right. \\
&-\frac{1}{44} \phi_{2} \mathscr{H}_{2} \phi_{2}+\frac{1}{66} \phi_{3} \mathscr{H}_{2} \phi_{1}+\frac{1}{22} \phi_{3} \mathscr{H}_{2} \mathscr{H}_{3} \phi_{3} \\
&+\sum_{k=3}^{\infty} c_{k}^{0} \phi_{k} \mathscr{H}_{k} \phi_{k}+\sum_{k=3}^{\infty} c_{k}^{*}\left(\phi_{k+1} \mathscr{H}_{k-1} \phi_{k-1}+\phi_{k-1} \mathscr{H}_{k+1} \phi_{k+1}\right) \\
&+\sum_{k=2}^{\infty} c_{k}^{+} \phi_{k} \mathscr{H}_{k+1}\left(\mathscr{H}_{k+2} \phi_{k+2}-\mathscr{H}_{k} \phi_{k}\right) \\
&\left.+\sum_{k=4}^{\infty} c_{k}^{-} \phi_{k} \mathscr{H}_{k-1}\left(\mathscr{H}_{k} \phi_{k}-\mathscr{H}_{k-2} \phi_{k-2}\right)\right]+\mathcal{O}\left(a^{6}+\sigma^{3}\right)
\end{aligned}
$$

where the constants

$$
\begin{aligned}
c_{k}^{0} & =\frac{1}{2\left(k^{2}-1\right)\left(2 k^{2}-2 k-1\right)\left(2 k^{2}+2 k-1\right)}, \\
c_{k}^{*} & =\frac{4 k^{4}-2 k^{2}+1}{12 k^{2}\left(2 k^{2}-2 k-1\right)\left(2 k^{2}+2 k-1\right)}, \\
c_{k}^{ \pm} & =\frac{k \pm 1}{4\left(2 k^{2} \pm 2 k-1\right)} .
\end{aligned}
$$

The model SDE (9) provides accurate simulations of the original SPDE (1), as the model is obtained through solving the SPDE to a specified order of its residual (Theorem 5). This model holds whether the forcing $\phi(x, t)$ is deterministic or stochastic, space-time correlated or independent, at each point in space and time. The infinite sums in the model SDE (9) represent the effects on the macroscale of the multitude of interactions among the microscale noises. In deterministic cases, Chicone \& Latushkin's theory of infinite-dimensional centre manifolds [12] supports (9) as a model of the deterministic but nonautonomous PDE (1). However, we proceed to consider exclusively the case when the applied forcing $\phi(x, t)$ is stochastic.

The outstanding challenge, with effects that are quadratic in the noise, is that we apparently cannot directly eliminate convolutions over the past history of the noise, such as $\phi_{1} \mathscr{H}_{2} \phi_{2}$, from the model.

\section{Stochastic resonance and deterministic drift}

Chao and Roberts $[\mathbf{1 0}, \mathbf{3 5}]$ argued that terms of $\mathcal{O}\left(\sigma^{2}\right)$ involving memory convolutions of the noise were effectively new drift and new noise terms when viewed over the long 
timescales of the relatively slow evolution of the model SDE (9). Analogously, Just et al. [19] argued that fast-time deterministic chaos appears as noise when viewed over long timescales. The arguments of Chao and Roberts $[\mathbf{1 0 , 3 5}]$ rely upon the noise being stochastic white noise. The model SDE (9) is a 'strong' model in that it faithfully tracks any given realisation of the original SPDE (Theorem 4). However, this section derives weak model SDEs, such as the SDE (4). The weak sense arises because we cannot know which realisation is being simulated, due to the effectively new noises on the macroscale. The memory convolutions are replaced through analysis of the long-time behaviour of their PDFs.

\subsection{Abandon fast-time convolutions}

The undesirable feature of the large-time model SDE (9) is the inescapable appearance in the model of fast-time convolutions in the quadratic noise terms, for example $\mathscr{H}_{2} \phi_{1}=$ $e^{-3 t} \star \phi_{1}$ and $\mathscr{H}_{2} \mathscr{H}_{3} \phi_{3}=e^{-3 t} \star e^{-8 t} \star \phi_{3}$. These require resolution of the fast-time response of the system to these fast-time dynamics in order to maintain fidelity with the original SPDE (1), and so they incongruously require small time-steps to find a supposedly slowly evolving amplitude $a$. However, maintaining fidelity with the full details of a white-noise source is a pyrrhic victory when all we are interested in is the relatively slow long-term dynamics. Instead, we need only those parts of the quadratic noise factors, such as $\phi_{1} \mathscr{H}_{2} \phi_{1}$ and $\phi_{1} \mathscr{H}_{2} \mathscr{H}_{3} \phi_{3}$, that over long timescales are firstly correlated with the other processes that appear, and are secondly independent of the other processes; these not only introduce factors in new independent noises into the model, but also introduce a deterministic drift due to stochastic resonance (as also noted by Drolet \& Vinal [14]).

To the order of accuracy of the strong model SDE (9), we need to understand the long-term effects of quadratic noises appearing in the form $\phi_{j} \mathscr{H}_{p} \phi_{i}$ and $\phi_{j} \mathscr{H}_{q} \mathscr{H}_{p} \phi_{i}$. These two sorts of terms appear in the right-hand side of the SDE (9) in the form $\dot{a}=\cdots \sigma^{2} c \phi_{j} \mathscr{H}_{p} \phi_{i} \cdots$, for example. Equivalently, we can rewrite this form as $d a=\cdots \sigma^{2} c \phi_{j} \mathscr{H}_{p} \phi_{i} d t \cdots$. In this latter form, we aim to replace such a quadratic noise term by a corresponding stochastic differential, so that $d a=\cdots \sigma^{2} c d y_{1} \cdots$ for some stochastic process $y_{1}$ with some drift and volatility: $d y_{1}=() d t+() d W$ for a Wiener process $W$. We work similarly for a noise term involving $\phi_{j} \mathscr{H}_{q} \mathscr{H}_{p} \phi_{i}$. Thus we must understand the long-term dynamics of stochastic processes $y_{1}$ and $y_{2}$ defined via the nonlinear SDEs

$$
\frac{d y_{1}}{d t}=\phi_{j} \mathscr{H}_{p} \phi_{i} \quad \text { and } \quad \frac{d y_{2}}{d t}=\phi_{j} \mathscr{H}_{q} \mathscr{H}_{p} \phi_{i} .
$$

\subsection{Canonical quadratic noise interactions}

To proceed following the argument put forward by Chao \& Roberts [10, $\$ 4.1]$, we name the two coloured noises that appear in the nonlinear terms (10): we define $z_{1}=\mathscr{H}_{p} \phi_{i}$ and $z_{2}=\mathscr{H}_{q} \mathscr{H}_{p} \phi_{i}$. From (6), these satisfy the SDES

$$
\frac{d z_{1}}{d t}=-\beta_{1} z_{1}+\phi_{i} \quad \text { and } \quad \frac{d z_{2}}{d t}=-\beta_{2} z_{2}+z_{1},
$$

where for this particular SPDE (1) the rates of decay are $\beta_{1}=p^{2}-1$ and $\beta_{2}=q^{2}-1$. Now put the SDES (10) and (11) together; we must understand the long-term properties of $y_{1}$ and $y_{2}$ governed by the coupled system of SDEs

$$
\begin{array}{ll}
\dot{y}_{1}=z_{1} \phi_{j}, & \dot{z}_{1}=-\beta_{1} z_{1}+\phi_{i}, \\
\dot{y}_{2}=z_{2} \phi_{j}, & \dot{z}_{2}=-\beta_{2} z_{2}+z_{1} .
\end{array}
$$


There are two cases to consider: when $i=j$ the two source noises $\phi_{i}$ and $\phi_{j}$ are identical; but when $i \neq j$ the two noise sources are independent. Understanding the SDEs (12) will help us replace memory convolutions in modelling general nonlinear stochastic processes, not just in the modelling of the SPDE (1).

\subsection{Use the Fokker-Planck equation}

Following Chao \& Roberts [10, 35] and analogously to Just et al. [19], we explore the long-term dynamics of the canonical quadratic system (12) via the Fokker-Planck equation for the joint PDF $P(\vec{y}, \vec{z}, t)$. Vanden-Eijnden [41] similarly uses the Kolmogorov forward equation to model the slow modes in SDEs. The canonical system (12) shows that, if we neglect the forcing $\phi_{i}$ and $\phi_{j}$, the $\vec{z}$ variables decay exponentially, whereas the $\vec{y}$ variables are constant. Consequently, upon retaining the forcing, over long times we expect the $\vec{z}$ variables to settle onto some quasi-stationary probability distribution (see, for example, [27]), whereas the $\vec{y}$ variables would evolve slowly. Thus we proceed to approximately factor the joint PDF into

$$
P(\vec{y}, \vec{z}, t) \approx p(\vec{y}, t) G_{0}(\vec{z}) .
$$

Here, $G_{0}(\vec{z})$ is some distribution to be determined, depending upon the coefficients $\vec{\beta}$. The quasi-PDF $p(\vec{y}, t)$ evolves slowly in time according to a PDE that we interpret as a FokkerPlanck equation for the long-term evolution of the interesting variables $\vec{y}(t)$.

We begin by analysing the Fokker-Planck equation for the joint PDF $P(\vec{y}, \vec{z}, t)$ of the canonical system (12). Recall that throughout we adopt the Stratonovich interpretation of SDEs; thus the Fokker-Planck equation of the SDEs (12) is

$$
\begin{aligned}
\frac{\partial P}{\partial t}= & \frac{\partial}{\partial z_{1}}\left(\beta_{1} z_{1} P\right)+\frac{\partial}{\partial z_{2}}\left[\left(\beta_{2} z_{2}-z_{1}\right) P\right]+\frac{1}{2} \frac{\partial^{2} P}{\partial z_{1}^{2}} \\
& +\frac{1}{2} s \sum_{k=1}^{2} \frac{\partial}{\partial y_{k}}\left(z_{k} \frac{\partial P}{\partial z_{1}}\right)+\frac{1}{2} \sum_{k, l=1}^{2} \frac{\partial}{\partial y_{k}}\left(z_{k} z_{l} \frac{\partial P}{\partial y_{l}}\right),
\end{aligned}
$$

where the parameter $s=1$ for the identical noise case $i=j$, whereas $s=0$ for the independent noise case $i \neq j$.

\subsection{A deterministic slow manifold captures the long-term dynamics}

The first line in the Fokker-Planck equation (14) represents all the rapidly dissipative processes: the terms of the form $\partial_{z_{k}}\left[\beta_{k} z_{k} P\right]$ 'move' probability density $P$ to the vicinity of $z_{k}=0$; this movement is balanced by the spread induced through the stochastic noise term $\frac{1}{2} P_{z_{1} z_{1}}$ and the forcing term $\partial_{z_{2}}\left[-z_{1} P\right]$. In contrast, the terms in the second line of the Fokker-Planck equation (14) describe the way that the PDF $P$ will slowly spread in the $y_{k}$ directions over long times. This strong disparity in timescales of the $\vec{y}$ and $\vec{z}$ evolution has led many (see, for example, $[\mathbf{1 4}, \mathbf{3 8}, \mathbf{1 9}]$ ) to the conditional factorisation (13). However, we systematically go further by appealing to deterministic centre manifold theory $[\mathbf{1 0}, \mathbf{3 4}, \mathbf{1 7}]$; alternatively, one can appeal to the SCM theory outlined in Appendix A but without any noise.

We treat the terms in the second line of the Fokker-Planck equation (14) as small perturbation terms by assuming that the structures in the $y_{k}$ variables are slowly varying; that is, we treat $\partial / \partial y_{k}$ as asymptotically 'small' parameters $[\mathbf{2 8 , 3 4 ]}$, as is appropriate over long times. Then 'linearly' (that is, upon ignoring the 'small' $\vec{y}$ derivative terms in the second 
line) the dynamics of the Fokker-Planck equation (14) are those of exponential attraction to a manifold of equilibria $P \propto G_{0}(\vec{z})$ at each $\vec{y}$; say the constant of proportionality is $p$. Theory for slow variations in space $[28,34]$ then assures us that a slow manifold exists for the Fokker-Planck equation (14), and that all dynamics (as it is a liner PDE) are exponentially quickly attracted to the dynamics on the slow manifold. The approximation theorem then assures us that the long-term dynamics of the joint PDF $P$, when the small terms in the second line of (14) are accounted for, may be expressed as a series in gradients in $\vec{y}$ of the slowly evolving $p(\vec{y}, t)$ : using $\vec{\nabla}$ for the vector gradient $\partial / \partial y_{k}$, we have the PDF

$$
P(\vec{y}, \vec{z}, t)=G_{0}(\vec{z}) p+\vec{G}_{1}(\vec{z}) \cdot \vec{\nabla} p+\mathbb{G}_{2}(\vec{z}): \vec{\nabla} \vec{\nabla} p+\cdots,
$$

where, instead of being constant, the quasi-conditional probability $p$ evolves slowly in time according to a series in gradients of $p$ in $\vec{y}$ of the Kramers-Moyal form (see, for example, $[26,25,40])$ :

$$
\frac{\partial p}{\partial t}=-\vec{U} \cdot \vec{\nabla} p+\mathbb{D}: \vec{\nabla} \vec{\nabla} p+\cdots
$$

In practice, we truncate this Kramers-Moyal expansion to include up to the second-order gradients in $\vec{y}$ for three reasons: firstly, the terms appearing explicitly in (16) form the lowest-order, structurally stable model for $p(\vec{y}, t)$; secondly, Pawula's theorem implies that any higher-order truncation may lead to negative probabilities; and thirdly, we interpret the second-order truncation of the Kramers-Moyal expansion (16) as a Fokker-Planck equation for the long-term evolution of the interesting $\vec{y}$ processes. Just et al. in [19, equation (11)] similarly truncate to second order. Deterministic centre manifold theory assures us that all solutions are attracted to the model (15)-(16); see, for example, [34, §2.2.2].

\subsection{Construct the long-term model}

The approximation theorem of centre manifolds (see, for example, [34, §2.2.3]) asserts that we simply substitute the ansatz (15)-(16) into the governing Fokker-Planck equation (14) and solve to reduce the residuals to some order of asymptotic error; then the slow-manifold model is constructed to the same order of accuracy. Here the order of accuracy is measured by the number of $\vec{y}$ gradients, $\vec{\nabla}$. Consequently, an error denoted as $\mathcal{O}\left(\vec{\nabla}^{q} p\right)$ denotes all terms of the form

$$
\partial^{q_{1}+q_{2}} p / \partial y_{1}^{q_{1}} \partial y_{2}^{q_{2}} \quad \text { for } q_{1}+q_{2} \geqslant q .
$$

For example, truncating (15)-(16) to the shown terms has errors $\mathcal{O}\left(\vec{\nabla}^{3} p\right)$. Computer algebra machinations [36, §2] driven by the residuals of the Fokker-Planck equation (14) readily find the coefficients of the slow-manifold model (15)-(16).

The computer algebra [36, §2] readily determines that the joint PDF of large-time solutions of the SDEs (12) form the slow manifold

$$
\begin{gathered}
P=A \exp \left\{-\left(\beta_{1}+\beta_{2}\right)\left[z_{1}^{2}-2 \beta_{2} z_{1} z_{2}+\beta_{2}\left(\beta_{1}+\beta_{2}\right) z_{2}^{2}\right]\right\} \\
\times\left\{p-s\left[z_{1}^{2}-2 \beta_{2} z_{1} z_{2}+2 \beta_{2}\left(\beta_{1}+\beta_{2}\right) z_{2}^{2}+B_{1}\right] \frac{\partial p}{\partial y_{1}}\right. \\
\left.-s\left[\left(\beta_{1}+\beta_{2}\right) z_{2}^{2}+B_{2}\right] \frac{\partial p}{\partial y_{2}}+\mathcal{O}\left(\vec{\nabla}^{2} p\right)\right\},
\end{gathered}
$$

for some normalisation constants $A, B_{1}$ and $B_{2}$. 
Simultaneously with finding the next-order corrections to this joint PDF, the computer algebra finds that the relatively slowly varying, quasi-conditional probability density $p$ evolves according to the Kramers-Moyal expansion

$$
\frac{\partial p}{\partial t}=-\frac{1}{2} s \frac{\partial p}{\partial y_{1}}+\mathbb{D}: \vec{\nabla} \vec{\nabla} p+\mathcal{O}\left(\vec{\nabla}^{3} p\right),
$$

where the constant diffusion matrix is

$$
\mathbb{D}=\frac{1}{4 \beta_{1}}\left[\begin{array}{cc}
1 & \frac{1}{\beta_{1}+\beta_{2}} \\
\frac{1}{\beta_{1}+\beta_{2}} & \frac{1}{\beta_{2}\left(\beta_{1}+\beta_{2}\right)}
\end{array}\right] .
$$

\subsection{Translate to a corresponding SDE}

We now interpret (17) as a Fokker-Planck equation. It corresponds to the coupled SDES

$$
\dot{y}_{1}=\frac{1}{2} s+\frac{\psi_{1}(t)}{\sqrt{2 \beta_{1}}} \quad \text { and } \quad \dot{y}_{2}=\frac{1}{\beta_{1}+\beta_{2}}\left(\frac{\psi_{1}(t)}{\sqrt{2 \beta_{1}}}+\frac{\psi_{2}(t)}{\sqrt{2 \beta_{2}}}\right) .
$$

Of course, there are many coupled SDEs whose Fokker-Planck equation is the PDE (17): the reason is there are many $2 \times 2$ volatility matrices $\mathbb{S}$ of coupled SDEs that give the same diffusivity matrix $\mathbb{D}=\frac{1}{2} \mathbb{S S}^{T}$; for example, Just et al. [19] choose $\mathbb{S}$ to be the positive definite, symmetric square root of the diffusivity matrix $2 \mathbb{D}$. For our purposes, any of the possible volatility matrices would suffice: in resorting to the Fokker-Planck equations we necessarily lose fidelity of paths, and now require only fidelity of distributions and correlations; as commented earlier, the result is a weak model, not a strong model. For simplicity, we obtain the form of the noise terms in (19) by the unique Cholesky factorisation of the diffusion matrix

$$
\mathbb{D}=\frac{1}{2} \mathbb{L} \mathbb{L}^{T}
$$

for matrix

$$
\mathbb{L}=\left[\begin{array}{cc}
\frac{1}{\sqrt{2 \beta_{1}}} & 0 \\
\frac{1}{\sqrt{2 \beta_{1}}\left(\beta_{1}+\beta_{2}\right)} & \frac{1}{\sqrt{2 \beta_{2}}\left(\beta_{1}+\beta_{2}\right)}
\end{array}\right] .
$$

Choosing the volatility matrix in the SDEs to be the lower triangular Cholesky matrix $\mathbb{L}$ ensures that nearly half the terms in the volatility matrix are zero, and it also ensures that when we go to higher-order convolutions of noise in Section 5, this $2 \times 2$ factorisation remains in the higher-order factorisations; see the $4 \times 4$ Cholesky matrix (28).

The processes $\psi_{i}(t)$ in (19) are new noises, independent of $\phi_{i}$ and $\phi_{j}$ over long timescales. Chao \& Roberts $[\mathbf{1 0}, \mathbf{3 5}]$ showed this independence numerically, and Appendix B presents an analysis supporting this independence. One remarkable feature to see in the SDEs (19) is that for the case of identical noise, $\phi_{i}=\phi_{j}$, in the case $s=1$, there is a mean drift of $\frac{1}{2}$ in the stochastic process $y_{1}$; there is no mean drift in any other process, nor in the other case, $s=0$.

One might query the role of the neglected terms in the Kramers-Moyal expansions of the PDF (15) and the supposed Fokker-Planck equation (17). In the joint PDF (15), the neglected $\mathcal{O}\left(\vec{\nabla}^{3} p\right)$ terms provide more details of the non-Gaussian structure of the joint 
PDF in the slowly evolving, long-time dynamics. The effects of the neglected $\mathcal{O}\left(\vec{\nabla}^{3} p\right)$ terms in (17) correspond to algebraically decaying departures from the second-order truncation that we interpret as a Fokker-Planck equation; Chao \& Roberts [10] demonstrated this algebraic decay to normality in some numerical simulations. (Chatwin [11] discussed this algebraic approach to normality in detail in the simpler situation of dispersion in a channel.) Such algebraically decaying transients may represent slow decay of non-Markovian effects among the $\vec{y}$ variables. However, the truncation (17) that we interpret as a Fokker-Planck equation is the lowest-order structurally stable model, and so it models the dynamics over the longest timescales.

\subsection{Temporarily truncate the noise to simplify discussion}

To simplify the detailed strong model SDE (9) further, we eliminate the nonlinear fast-time memory convolutions to deduce a model that is nearly as simple as the SDE (4). However, dealing with the infinite sums in the strong model SDE (9) is confusing when the focus is on transforming the nonlinear fast-time convolutions. Thus temporarily we discuss the case when the applied spatio-temporal noise (2) is truncated to the first three modes:

$$
\phi=\sum_{k=1}^{3} \phi_{k}(t) \sin k x \text {. }
$$

Just these three noise components have a range of interactions that are representative of the noise interactions appearing in the SDE (9) to the order of accuracy reported here, and for the nonlinearity of this SPDE. Thus, to focus on the transformations of the noise, we temporarily consider the strong model SDE (9) with the truncated noise; that is,

$$
\begin{aligned}
\dot{a}= & -\frac{1}{12} a^{3}-\frac{7}{3456} a^{5} \\
& +\sigma \phi_{1}+\frac{1}{6} a \sigma \phi_{2}+a^{2} \sigma\left(\frac{1}{18} \phi_{1}+\frac{1}{96} \phi_{3}\right)+a^{3} \sigma \frac{1}{54} \phi_{2} \\
& +\sigma^{2}\left(\frac{1}{6} \phi_{1} \mathscr{H}_{2} \phi_{2}+\frac{1}{22} \phi_{3} \mathscr{H}_{2} \phi_{2}+\frac{1}{22} \phi_{2} \mathscr{H}_{3} \phi_{3}\right) \\
+ & a \sigma^{2}\left[\frac{1}{18} \phi_{1} \mathscr{H}_{2} \phi_{1}-\frac{1}{44} \phi_{2} \mathscr{H}_{2} \phi_{2}+\frac{1}{4048} \phi_{3} \mathscr{H}_{3} \phi_{3}+\frac{19}{528} \phi_{1} \mathscr{H}_{3} \phi_{3}\right. \\
& +\frac{1}{66} \phi_{3} \mathscr{H}_{2} \phi_{1}-\frac{3}{44} \phi_{2} \mathscr{H}_{3} \mathscr{H}_{2} \phi_{2}+\frac{1}{6} \phi_{1} \mathscr{H}_{2} \mathscr{H}_{3} \phi_{3}+\frac{1}{22} \phi_{3} \mathscr{H}_{2} \mathscr{H}_{3} \phi_{3} \\
& \left.-\frac{1}{23} \phi_{3} \mathscr{H}_{4} \mathscr{H}_{3} \phi_{3}\right]+\mathcal{O}\left(a^{6}+\sigma^{3}\right) .
\end{aligned}
$$

\subsection{Transform the strong model (21) to be usefully weak.}

The quadratic noises in the strong model SDE (21) involve the convolutions $\mathscr{H}_{2}, \mathscr{H}_{3}$ and $\mathscr{H}_{4}$, which have corresponding decay rates $\beta$ of 3,8 and 15 respectively. Thus from the various instances of (19), to obtain a model for long timescales we replace the quadratic noises as follows: 


$$
\begin{aligned}
\phi_{1} \mathscr{H}_{2} \phi_{2} & \mapsto \frac{\psi_{1}}{\sqrt{6}}, \\
\phi_{3} \mathcal{H}_{2} \phi_{2} & \mapsto \frac{\psi_{2}}{\sqrt{6}}, \\
\phi_{2} \mathscr{H}_{3} \phi_{3} & \mapsto \frac{\psi_{3}}{4}, \\
\phi_{1} \mathcal{H}_{2} \phi_{1} & \mapsto \frac{1}{2}+\frac{\psi_{4}}{\sqrt{6}}, \\
\phi_{2} \mathscr{H}_{2} \phi_{2} & \mapsto \frac{1}{2}+\frac{\psi_{5}}{\sqrt{6}}, \\
\phi_{3} \mathscr{H}_{3} \phi_{3} & \mapsto \frac{1}{2}+\frac{\psi_{6}}{4}, \\
\phi_{1} \mathcal{H}_{3} \phi_{3} & \mapsto \frac{\psi_{7}}{4}, \\
\phi_{3} \mathcal{H}_{2} \phi_{1} & \mapsto \frac{\psi_{8}}{\sqrt{6}}, \\
\phi_{2} \mathscr{H}_{3} \mathscr{H}_{2} \phi_{2} & \mapsto \frac{\psi_{5}}{11 \sqrt{6}}+\frac{\psi_{9}}{44}, \\
\phi_{1} \mathscr{H}_{2} \mathscr{H}_{3} \phi_{3} & \mapsto \frac{\psi_{7}}{44}+\frac{\psi_{10}}{11 \sqrt{6},} \\
\phi_{3} \mathscr{H}_{2} \mathscr{H}_{3} \phi_{3} & \mapsto \frac{\psi_{6}}{44}+\frac{\psi_{11}}{11 \sqrt{6}}, \\
\phi_{3} \mathscr{H}_{4} \mathscr{H}_{3} \phi_{3} & \mapsto \frac{\psi_{6}}{92}+\frac{\psi_{12}}{23 \sqrt{30}},
\end{aligned}
$$

where $\psi_{1}, \ldots, \psi_{12}$ are independent white noises (that is, derivatives of independent Wiener processes). Thus we transform the strong model SDE (21) to the weak model SDE

$$
\begin{aligned}
\dot{a}= & -\frac{1}{12} a^{3}-\frac{7}{3456} a^{5} \\
& +\sigma \phi_{1}+\frac{1}{6} a \sigma \phi_{2}+a^{2} \sigma\left(\frac{1}{18} \phi_{1}+\frac{1}{96} \phi_{3}\right)+a^{3} \sigma \frac{1}{54} \phi_{2} \\
& +\sigma^{2}\left[\frac{\psi_{1}}{6 \sqrt{6}}+\frac{\psi_{2}}{22 \sqrt{6}}+\frac{\psi_{3}}{88}\right]+\frac{1}{2} a \sigma^{2}\left(\frac{1}{18}-\frac{1}{44}+\frac{1}{4048}\right) \\
& +a \sigma^{2}\left[\frac{\psi_{4}}{18 \sqrt{6}}-\frac{7 \psi_{5}}{242 \sqrt{6}}+\frac{2549 \psi_{6}}{4096576}+\frac{9 \psi_{7}}{704}+\frac{\psi_{8}}{66 \sqrt{6}}-\frac{3 \psi_{9}}{1936}\right. \\
& \left.+\frac{\psi_{10}}{66 \sqrt{6}}+\frac{\psi_{11}}{242 \sqrt{6}}-\frac{\psi_{12}}{529 \sqrt{30}}\right]+\mathcal{O}\left(a^{6}+\sigma^{3}\right) .
\end{aligned}
$$

Here the new noise processes $\psi_{k}$ appear in only two different combinations (the bracketed terms). Thus we do not need to use them individually, only their combined effect. Combining the new noise processes into two effective new noise processes $\Phi_{1}$ and $\Phi_{2}$ (see $[36, \S 1.5]$ ), 
we see that the weak model (22) vastly simplifies to the weak model SDE

$$
\begin{aligned}
\dot{a}= & 0.01654 \sigma^{2} a-\frac{1}{12} a^{3}-\frac{7}{3456} a^{5} \\
& +\sigma \phi_{1}+\frac{1}{6} a \sigma \phi_{2}+a^{2} \sigma\left(\frac{1}{18} \phi_{1}+\frac{1}{96} \phi_{3}\right)+a^{3} \sigma \frac{1}{54} \phi_{2} \\
& +0.07144 \sigma^{2} \Phi_{1}+0.02999 \sigma^{2} a \Phi_{2}+\mathcal{O}\left(a^{6}+\sigma^{3}\right) .
\end{aligned}
$$

(The combinations $\sigma \phi_{1}+0.07144 \sigma^{2} \Phi_{1}$ and $\frac{1}{6} a \sigma \phi_{2}+0.02999 \sigma^{2} a \Phi_{2}$ in (23) could be combined, but then one must be careful with the correlations with the other noise terms on the second line of (23).) The Stratonovich model SDE (23) is a weak model of the original Stratonovich SPDE (1) because we have replaced detailed knowledge of the interactions of rapid microscale noise, seen in the convolutions of the strong model SDE (21), by their longtimescale statistics. Similarly, Just et al. [19] replaced detailed knowledge of rapid chaos by its long-timescale statistics. Vanden-Eijnden [41] comments that stronger results can be obtained. However, resolving rapid fluctuations seems futile when they are stochastic, as required for this section, because describing them as stochastic is an admission that we do not know their detail anyway. The weak model SDE (23) is useful because it only resolves long-timescale dynamics and hence, for example, we are empowered to efficiently solve the SDE with numerical schemes using large time-steps.

Furthermore, however, we readily discover crucial stability information in the weak model SDE (23). The quadratic interactions of noise processes, through stochastic resonance, generate the mean effect term $0.01654 \sigma^{2} a$. As it is a term that is linear in $a$ with positive coefficient $0.01654 \sigma^{2}$, this term destabilises the origin. Roberts [35] demonstrated in numerical simulations how the same term in $\sigma^{2} a$, but with a negative coefficient, stabilises the origin as expected. Thus we are empowered here by our analysis to predict instead that the stochastic solutions of the SPDE (1) will linger about (and make noise-induced transitions between) two fixed points obtained from the deterministic part of the model SDE (23), namely $u \approx a \sin x$ for amplitudes $a \approx \pm 0.45 \sigma$.

\subsection{Return to the full spectrum of noise}

Now we deal with the full complexity of the infinite sums of nonlinear noise interactions in the strong model SDE (9). First, we obtain the exact numerical coefficient for the stochastic resonance term $\sigma^{2} a$ for the full spectrum of noise through the infinite sum

$$
\sum_{k=3}^{\infty} c_{k}^{0} \phi_{k} \mathscr{H}_{k} \phi_{k}
$$

Terms of this form are the only ones contributing to this stochastic resonance. The exact numerical coefficient is thus

$$
\frac{1}{2}\left(\frac{1}{18}-\frac{1}{44}+\sum_{k=3}^{\infty} c_{k}^{0}\right)=0.016563
$$

to five significant digits. Curiously, in this problem, it is only the $\phi_{2} \sin 2 x$ component of the noise that acts to stabilise $u=0$ through its negative contribution to this sum; as explored in [35], all other noise components act to destabilise $u=0$ through their positive contribution. 
Second, and similarly, the other infinite sums over the noise components in the strong model SDE (9) modify the coefficients in the weak model SDE (23). However, as for the stochastic resonance term, the modification to the coefficients is not large: the plain $\sigma^{2}$ term from the third line of (9) has coefficients $\sim 1 / k^{2}$ (as $k \rightarrow \infty$ ) but $\phi_{k \pm 1} \mathscr{H}_{k} \phi_{k} \sim 1 / k$ (from (19) and that $\beta \sim k^{2}$ ), so that the terms in the sum are $\sim 1 / k^{3}$; similarly, the infinite sums in lines 6-8 of (9) have terms $\sim 1 / k^{4}$ or smaller. Further, when combining the infinitely many new noise terms in the analogue of (22) to find the exact version of the weak model SDE (23), the coefficients are the root-sum-squares of the coefficients of the new noise processes in the infinite sums; thus the terms $\mathcal{O}\left(1 / k^{3}\right)$ and $\mathcal{O}\left(1 / k^{4}\right)$ in the sums are terms of relative magnitude $\mathcal{O}\left(1 / k^{6}\right)$ and $\mathcal{O}\left(1 / k^{8}\right)$. Thus the infinite sums are accurately truncated after relatively few terms. Indeed, computer algebra [36, §1.5] demonstrates that at most ten terms in these sums determine the coefficients of the weak model SDE correct to five significant digits, namely

$$
\begin{aligned}
\dot{a}= & 0.016563 \sigma^{2} a-\frac{1}{12} a^{3}-\frac{7}{3456} a^{5} \\
& +\sigma \phi_{1}+\frac{1}{6} a \sigma \phi_{2}+a^{2} \sigma\left(\frac{1}{18} \phi_{1}+\frac{1}{96} \phi_{3}\right)+a^{3} \sigma \frac{1}{54} \phi_{2} \\
& +0.071843 \sigma^{2} \Phi_{1}+0.030368 \sigma^{2} a \Phi_{2}+\mathcal{O}\left(a^{6}+\sigma^{3}\right) .
\end{aligned}
$$

The earlier weak model SDE (23) with just the three coarsest microscale noise processes has coefficients correct to about $1 \%$ when compared to the model SDE (24) for the full spectrum of microscale noise.

\section{Quadratic noise interactions with up to four convolutions}

The strong model SDE (9) resolves the quadratic noise interactions affecting the terms in $\sigma^{2}$ and $\sigma^{2} a$. If we were to seek quadratic noise interactions affecting terms of higher order in the amplitude $a$, such as terms in $\sigma^{2} a^{2}$ and $\sigma^{2} a^{3}$, then we would face more convolutions of the noise: $\phi_{j} \mathscr{H}_{s} \mathscr{H}_{r} \mathscr{H}_{q} \mathscr{H}_{p} \phi_{i}$, for example. At such higher orders in a strong model, the infinite sums over the noise modes are considerably more complicated. Although such complication may be difficult to handle, the techniques for constructing a strong model are routine, as established in Sections 2 and 3. However, any useful weak model SDE of higher order in amplitude $a$ requires us to establish the correlations between noise processes with more convolutions than the two convolutions of equation (10). This section extends the analysis to four convolutions of the noise processes, to empower others to analyse to higher order in amplitude $a$ when needed in other problems.

To cater for more noise convolutions, we extend the canonical system of noise interactions, the SDEs (12). For up to $n$ convolutions of noise processes, we extend (12) to the system of SDES

$$
\begin{array}{cc}
\dot{y}_{1}=z_{1} \phi_{j}, & \dot{z}_{1}=-\beta_{1} z_{1}+\phi_{i}, \\
\dot{y}_{2}=z_{2} \phi_{j}, & \dot{z}_{2}=-\beta_{2} z_{2}+z_{1}, \\
\vdots & \vdots \\
\dot{y}_{n}=z_{n} \phi_{j}, & \dot{z}_{n}=-\beta_{n} z_{n}+z_{n-1} .
\end{array}
$$

Recall that the constants $\beta_{k}$ appearing here are just the decay rates of various of the fundamental modes of the linearised SPDE. Thus the results of this section apply to the modelling of general SDEs and SPDEs by appropriately setting the decay rates $\beta_{k}$. 
Consider the Fokker-Planck equation for the joint PDF $P(\vec{y}, \vec{z}, t)$ of the canonical system of SDEs (25). It is a straightforward extension of the Fokker-Planck equation (14). In the Stratonovich interpretation of SDEs, the Fokker-Planck equation is

$$
\begin{aligned}
\frac{\partial P}{\partial t}= & \frac{\partial}{\partial z_{1}}\left(\beta_{1} z_{1} P\right)+\sum_{k=2}^{n} \frac{\partial}{\partial z_{k}}\left[\left(\beta_{k} z_{k}-z_{k-1}\right) P\right]+\frac{1}{2} \frac{\partial^{2} P}{\partial z_{1}^{2}} \\
& +\frac{1}{2} s \sum_{k=1}^{n} \frac{\partial}{\partial y_{k}}\left(z_{k} \frac{\partial P}{\partial z_{1}}\right)+\frac{1}{2} \sum_{k, l=1}^{n} \frac{\partial}{\partial y_{k}}\left(z_{k} z_{l} \frac{\partial P}{\partial y_{l}}\right) .
\end{aligned}
$$

Using the same arguments as in Section 4, and treating $y_{k}$ derivatives as asymptotically small parameters, this Fokker-Planck equation has a slow manifold, that is exponentially quickly attractive, and may be constructed by making the residual of the Fokker-Planck equation (26) zero to some order. For a given number of convolutions $n$, computer algebra [36, §2] readily derives the terms in the slow-manifold model (15)-(16). For example, it appears that the leading-order Gaussian can be written in terms of a sum of squares as $G_{0}=A \exp \left(-\sum_{k=1}^{n} \beta_{k} \zeta_{k}^{2}\right)$, where

$$
\begin{aligned}
\zeta_{1}=z_{1}, \\
\zeta_{2}=z_{1}-\left(\beta_{1}+\beta_{2}\right) z_{2}, \\
\zeta_{3}=z_{1}-\left(\beta_{1}+2 \beta_{2}+\beta_{3}\right) z_{2}+\left(\beta_{1} \beta_{2}+\left(\beta_{1}+\beta_{2}+\beta_{3}\right) \beta_{3}\right) z_{3}, \\
\zeta_{4}=z_{1}-\left(\beta_{1}+2 \beta_{2}+2 \beta_{3}+\beta_{4}\right) z_{2} \\
\quad+\left(\beta_{1} \beta_{2}+\left(2 \beta_{1}+2 \beta_{2}+2 \beta_{3}+\beta_{4}\right) \beta_{3}+\left(\beta_{1}+\beta_{2}+\beta_{3}+\beta_{4}\right) \beta_{4}\right) z_{3} \\
\quad-\left(\beta_{1} \beta_{2} \beta_{3}+\left(\beta_{1} \beta_{2}+\beta_{1} \beta_{3}+\beta_{2} \beta_{3}\right) \beta_{4}+\left(\beta_{1}+\beta_{2}+\beta_{3}+\beta_{4}\right) \beta_{4}^{2}\right) z_{4} .
\end{aligned}
$$

However, using this algorithm to determine terms in $\vec{\nabla} p$ and $\vec{\nabla} \vec{\nabla} p$ requires more computer memory and time than I currently have available for anything more than the case of $n=3$ general noise convolutions with $\beta_{k}$ as variable parameters. For the accessible $n=3$ case, we find that the relatively slowly varying, quasi-conditional probability density $p$ evolves according to the Fokker-Planck-like PDE (17) but now the $3 \times 3$ diffusion matrix has entries

$$
\begin{aligned}
& \mathbb{D}_{11}=\frac{1}{4 \beta_{1}}, \\
& \mathbb{D}_{12}=\mathbb{D}_{21}=\frac{1}{4 \beta_{1}\left(\beta_{1}+\beta_{2}\right)}, \\
& \mathbb{D}_{22}=\frac{1}{4 \beta_{1} \beta_{2}\left(\beta_{1}+\beta_{2}\right)}, \\
& \mathbb{D}_{13}=\mathbb{D}_{31}=\frac{1}{4 \beta_{1}\left(\beta_{1}+\beta_{2}\right)\left(\beta_{1}+\beta_{3}\right)}, \\
& \mathbb{D}_{23}=\mathbb{D}_{32}=\frac{\beta_{1}+\beta_{2}+\beta_{3}}{4 \beta_{1} \beta_{2}\left(\beta_{1}+\beta_{2}\right)\left(\beta_{1}+\beta_{3}\right)\left(\beta_{2}+\beta_{3}\right)}, \\
& \mathbb{D}_{33}=\frac{\beta_{1}+\beta_{2}+\beta_{3}}{4 \beta_{1} \beta_{2} \beta_{3}\left(\beta_{1}+\beta_{2}\right)\left(\beta_{1}+\beta_{3}\right)\left(\beta_{2}+\beta_{3}\right)} .
\end{aligned}
$$

The $2 \times 2$ upper-left block is reassuringly identical to the earlier diffusion matrix (18). Fortunately, the alternative derivation in Appendix B of the diffusion matrix $\mathbb{D}$ is significantly more efficient. Computing the $4 \times 4$ diffusion matrix, I find the expressions extremely complicated and apparently not worth recording. 
However, the Cholesky factorisation is accessible. Recall that to interpret the PDE (17) as a Fokker-Planck equation of some system of SDEs, we desire the Cholesky factorisation of the diffusion matrix. The Cholesky factorisation here is $\mathbb{D}=\frac{1}{2} \mathbb{L} \mathbb{L}^{T}$ for the lower triangular matrix $\mathbb{L}$ with the following non-zero entries:

$$
\begin{aligned}
& \mathbb{L}_{11}=\frac{1}{\sqrt{2 \beta_{1}}} \\
& \mathbb{L}_{21}=\frac{1}{\sqrt{2 \beta_{1}}\left(\beta_{1}+\beta_{2}\right)}, \\
& \mathbb{L}_{22}=\frac{1}{\sqrt{2 \beta_{2}}\left(\beta_{1}+\beta_{2}\right)}, \\
& \mathbb{L}_{31}=\frac{1}{\sqrt{2 \beta_{1}}\left(\beta_{1}+\beta_{2}\right)\left(\beta_{1}+\beta_{3}\right)}, \\
& \mathbb{L}_{32}=\frac{1}{\sqrt{2 \beta_{2}}\left(\beta_{1}+\beta_{3}\right)}\left[\frac{1}{\beta_{1}+\beta_{2}}+\frac{1}{\beta_{2}+\beta_{3}}\right], \\
& \mathbb{L}_{33}=\frac{1}{\sqrt{2 \beta_{3}}\left(\beta_{2}+\beta_{3}\right)\left(\beta_{1}+\beta_{3}\right)}, \\
& \mathbb{L}_{41}=\frac{1}{\sqrt{2 \beta_{1}}\left(\beta_{1}+\beta_{2}\right)\left(\beta_{1}+\beta_{3}\right)\left(\beta_{1}+\beta_{4}\right)}, \\
& \mathbb{L}_{42}=\frac{1}{\sqrt{2 \beta_{2}}\left(\beta_{1}+\beta_{3}\right)}\left[\frac{1}{\left(\beta_{2}+\beta_{3}\right)\left(\beta_{2}+\beta_{4}\right)}+\frac{1}{\left(\beta_{1}+\beta_{4}\right)\left(\beta_{2}+\beta_{4}\right)}\right. \\
& \left.+\frac{1}{\left(\beta_{1}+\beta_{2}\right)\left(\beta_{1}+\beta_{4}\right)}\right] \text {, } \\
& \mathbb{L}_{43}=\frac{1}{\sqrt{2 \beta_{3}}\left(\beta_{2}+\beta_{4}\right)}\left[\frac{1}{\left(\beta_{1}+\beta_{3}\right)\left(\beta_{2}+\beta_{3}\right)}+\frac{1}{\left(\beta_{1}+\beta_{4}\right)\left(\beta_{3}+\beta_{4}\right)}\right. \\
& \left.+\frac{1}{\left(\beta_{1}+\beta_{3}\right)\left(\beta_{1}+\beta_{4}\right)}\right] \text {, } \\
& \mathbb{L}_{44}=\frac{1}{\sqrt{2 \beta_{4}}\left(\beta_{1}+\beta_{4}\right)\left(\beta_{2}+\beta_{4}\right)\left(\beta_{3}+\beta_{4}\right)} .
\end{aligned}
$$

There are some intriguing hints of relatively simple patterns developing in the entries of $\mathbb{L}$. Maybe an even more direct derivation via a change in measure for the hierarchy (25) could be exploited to derive general formulae for more convolutions of noise. The upper-left entries are also reassuringly identical to the earlier $2 \times 2$ case (20). These formulae empower us to transform general quadratic nonlinear combinations of noise processes into effectively new and independent noise processes for the long-time dynamics of quite general SDEs and SPDES.

\section{Conclusion}

The crucial virtue of the weak model SDEs (4) and (24), as also recognised by Just et al. [19], is that we may accurately take long time-steps as all the fast-time dynamics have been eliminated. The critical innovation here is that we have demonstrated, via the particular example SPDE (1), how it is feasible to analyse the net effect of a multitude of independent, subgrid, microscale, stochastic effects. Three important results follow: 
- we can remove all memory convolutions (convolutions) from the model SDE;

- nonlinear effects quadratic in the noise processes effectively generate a mean drift; and

- nonlinear effects quadratic in the noise processes effectively abstract new noise processes from the seething microscale interactions.

The general formulae in Section 5, together with the iterative construction of slow manifold models [29], empower us to model quite generic SPDEs.

My aim is to construct sound, discrete models of SPDEs. Here we have treated the whole domain of the SPDE as one element. The next step in the development of this approach to creating good discretisations of SPDEs is to divide the spatial domain into finite-sized elements and then systematically to analyse their subgrid processes together with the appropriate physical coupling between the elements, as we have instigated for deterministic PDEs (see, for example, $[30,32,24]$ ).

\section{Appendix A. Summary of stochastic centre manifold theory}

For the convenience of the reader, this section records crucial parts of the stochastic centre manifold theory of Boxler [7]. This theory underpins the analysis of Sections 2-5. As Arnold comments [1, p. 317], the theory is 'inevitably very technical'.

The italic text, such as that in the theorems presented here, is taken from Boxler [7] with minor modifications. Text in roman font denotes my simplified interpretation.

Extant SCM theory addresses a formal SDE equation in $d$ dimensions: $\dot{x}=F(\omega, x)$ where $\omega$ parametrises all the different realisations of the 'noise'. However, theory is almost always better expressed in terms of integrals. Hence, the theory is phrased in terms of the formal integral of the stochastic differential equation starting from a generic initial condition, $x=x_{0}$ at $t=0$. Thus the stochastic ensemble trajectory/orbit $x=\varphi\left(t, \omega, x_{0}\right)$ is defined to represent a stochastic/random dynamical system (RDS); see also [1, §1.1].

DeFINITION 1 (RDS [7, Definition 2.1]). Let $(\Omega, \mathcal{F}, P)$ be a probability space, and let $\vartheta_{t}$ be a group of bimeasurable measure-preserving bijections on $\Omega$. A map $\varphi: \mathbb{R} \times \Omega \times \mathbb{R}^{d} \rightarrow$ $\mathbb{R}^{d},(t, \omega, x) \rightarrow \varphi(t, \omega, x)$, is called a random dynamical system of $\mathrm{C}^{k}$-diffeomorphisms $(k \geqslant 1)$ on $\mathbb{R}^{d}$ over the flow $\left\{\vartheta_{t}: t \in \mathbb{R}\right\}$ if the following properties are satisfied.

1. $\varphi(t, \cdot, x)$ is $\mathcal{F}, \mathcal{B}\left(\mathbb{R}^{d}\right)$-measurable for any $t \in \mathbb{R}, x \in \mathbb{R}^{d}$.

2. There is a $\vartheta_{t}$-invariant set $\Omega_{0} \subset \Omega, P\left(\Omega_{0}\right)=1$, such that for all $\omega \in \Omega_{0}$ :

(a) $\varphi(t, \omega, x)$ is continuous in $(t, x) \in \mathbb{R} \times \mathbb{R}^{d}$;

(b) $\varphi(t, \omega, \cdot)$ is a $\mathrm{C}^{k}$-diffeomorphism for any $t \in \mathbb{R}$ (herein, $\mathrm{C}^{k, 1}$ means that $D^{k} \varphi(t, \omega, \cdot)$ satisfies a global Lipschitz condition $)$;

(c) $\varphi(t+s, \omega, x)=\varphi\left(s, \vartheta_{t} \omega, \cdot\right) \circ \varphi(t, \omega, x)$ for any $t, s \in \mathbb{R}, x \in \mathbb{R}^{d}$ (cocycle property).

This last cocycle property generalises the semigroup property of integrals of deterministic differential equations. The shift in time $\vartheta_{t}$ of the realisation $\omega$ accommodates the change in origin of time for the evolution over a time $t$ from time 0 , to the evolution over a time $s$ from time $t$.

In a considerable simplification, by transforming to a moving coordinate system attached to the orbit $\varphi(t, \omega, x) \ldots$ without loss of generality the random dynamical system $\varphi$ may be assumed to have an equilibrium point at the origin $x=0$; see [7, Proposition 4.1]. 
Based about the dynamics near the origin, we partition the dynamics into slow centre modes, exponentially decaying modes and exponentially growing modes. Oseledec's Multiplicative Ergodic Theorem asserts that there is a decomposition of the state space $\mathbb{R}^{d}$ into a finite number of Oseledec spaces (analogous to eigenspaces), each Oseledec space characterised by a different (real) Lyapunov exponent $\lambda_{i}$ obtained from the linearised dynamics, and the Oseledec spaces collectively span the state space $\mathbb{R}^{d}$. The bounds $\lambda_{s}=\max _{\lambda_{i}<0} \lambda_{i}$ and $\lambda_{u}=\min _{\lambda_{i}>0} \lambda_{i}$ are crucial in characterising the timescale separation between the centre, decaying and growing modes. Then $E_{c}(\omega), E_{s}(\omega)$ and $E_{u}(\omega)$ denote the spaces spanned by those Oseledec spaces, respectively corresponding to zero, negative and positive Lyapunov exponent. Subscripts $c, s$ and $u$ denote projections onto the subspaces $E_{c}(\omega)$, $E_{s}(\omega)$ and $E_{u}(\omega)$. For example, we may write $x=\left(x_{c}, x_{s}, x_{u}\right)$. The SCM is a nonlinear counterpart of the centre Oseledec space $E_{c}(\omega)$.

We distinguish the linear and nonlinear parts of the RDS by the decomposition $\varphi(t, \omega, x)=$ $T \varphi(t, \omega, 0) x+\Phi(t, \omega, x)$. The theory applies only to RDSs with a nonlinear part that has sufficiently small derivatives $\left\|D^{j} \Phi(t, \omega, x)\right\|$ for $j=0, \ldots, k$ and a Lipschitz condition on $D^{k} \Phi(t, \omega, x)$. Denote this class of RDSs by $N L_{\varepsilon_{0}, \ldots, \varepsilon_{k}}$. The following existence theorem then applies.

THeOREM 2 (Existence [7, Theorem 5.1]). There is a constant $L_{0}$ such that for any $L$, $0<L \leqslant L_{0}$, and any $\beta, 0<\beta<\frac{1}{4} \min \left(-\lambda_{s}, \lambda_{u}\right)$, there are constants $\varepsilon_{0}(L) \geqslant$ $\cdots \geqslant \varepsilon_{k}(L)>0$, which depend on $\beta$ and for any random dynamical system of class $N L_{\varepsilon_{0}(L), \ldots, \varepsilon_{k}(L)}$ which consists of $\mathrm{C}^{k, 1}$-diffeomorphisms there exists a global stochastic $\mathrm{C}^{k, 1}$-centre manifold for $P$-a.a. $\omega \in \Omega$. It may be written in the form

$$
M(\omega)=\left\{\left(x_{s}, h_{s}\left(\omega, x_{s}\right), h_{u}\left(\omega, x_{s}\right)\right) \mid x_{s} \in E_{c}(\omega)\right\}
$$

with a function $h \in A_{k}(L)$.

The space $A_{k}(L)$ mentioned in this theorem denotes strictly nonlinear stochastic functions, $h(\omega, 0)=0$ and $D^{1} h(\omega, 0)=0$, which are smooth enough; that is, for all $x, \tilde{x} \in E_{c}(\omega)$, we have $\left|D^{j} h(\omega, x)\right| \leqslant \frac{1}{2} L$ for all $j=0, \ldots, k$ and the Lipshitz condition $\left|D^{k} h(\omega, x)-D^{k} h(\omega, \tilde{x})\right| \leqslant \frac{1}{2} L|x-\tilde{x}|$. That is, the SCM $M(\omega)$ is written as a graph over the centre Oseledec space $E_{c}(\omega)$

This global existence theorem is very restrictive. Much more useful in practice is the existence of a local centre manifold based upon the linearisation at an equilibrium. Further, when the deterministic part of the linearisation has eigenvalues that are all zero (the imaginary part is zero), then we may call the centre manifold by the more informative name of 'slow manifold', as is done in this paper.

TheOREM 3 (LOCAL EXISTENCE [7, Theorem 6.1]). Let $\varphi$ be a random dynamical system on $\mathbb{R}^{d}$ which consists of $\mathrm{C}^{k}$-diffeomorphisms $(k \geqslant 2)$ and satisfies the assumptions of Definition 1. If one of the Lyapunov exponents of the linearization vanishes, then there will be a local stochastic $\mathrm{C}^{k-1}$-centre manifold for $\varphi$.

For modelling, we require the dynamics to settle onto a low-dimensional manifold so that the dynamics are described by the evolution of the relatively few variables parametrising the manifold. SCM theory assures this when there are no growing modes; that is, when $E_{u}(\omega)=\varnothing$. Further, the theory asserts that the evolution of the orbits approaching the SCM are the same, to an exponentially decaying difference, as those orbits actually on the SCM. For deterministic dynamics this property is sometimes called 'asymptotic completeness' [37]. Thus the following theorem guarantees the relevance of the dynamics on the SCM $M(\omega)$. 
TheOREM 4 (RElEVAnCE [7, Theorem 7.1]). Let $M(\omega)$ be the stochastic centre manifold of Theorem 2. If all Lyapunov exponents are less than or equal to 0 , then there is a map $c_{s}: \mathbb{R}^{+} \rightarrow(0, \infty)$ satisfying $\lim _{t \rightarrow \infty}(1 / t) \log c_{S}(t)<0$ such that for any initial values $x_{c} \in E_{c}(\omega)$ and $x_{s} \in E_{s}(\omega)$ :

$$
\left|\varphi\left(t, \omega, x_{c}, x_{s}\right)-h\left[\vartheta_{t} \omega, \varphi\left(t, \omega, x_{c}, x_{s}\right)\right]\right|_{\vartheta_{t} \omega}^{s} \leqslant c_{s}(t)\left|x_{s}-h_{s}\left(\omega, x_{c}\right)\right|_{\omega}^{s}
$$

for any $t \geqslant 0$ almost surely.

This inequality asserts that the difference between orbits on and off $M(\omega)$ is bounded by the initial difference and an essentially exponentially decaying function $c_{S}(t)$. Thus orbits on $M(\omega)$ model the dynamics from a wide variety of initial conditions.

Lastly, we construct SCMs by approximately satisfying the governing SDEs. The following theorem asserts that the error in the SCM is of the same order as the residual of the governing equations of the random dynamical system. Consider that $x=\eta\left(\omega, x_{c}\right)$ approximates the actual SCM $x=h\left(\omega, x_{c}\right)$, and let the operator $V$ compute the residual of the governing equations, so that $V \eta$ is the residual for the approximation $\eta$. Then the following theorem underpins the orders of errors given throughout this paper.

Theorem 5 (Approximation [7, Theorem 8.1]). For a given $\eta \in A_{k}(L)$, assume that $(V \eta)\left(\omega, x_{c}\right)=\mathcal{O}\left(\left\|x_{c}\right\|^{q}\right)$ for some $q>1$, almost all $\omega \in \Omega$ and all $x_{c} \in E_{c}(\omega)$ sufficiently small. Then we obtain:

$$
\left\|h\left(\omega, x_{c}\right)-\eta\left(\omega, x_{c}\right)\right\|=\mathcal{O}\left(\left\|x_{c}\right\|^{q}\right) .
$$

Finally, note that the theory of stochastic normal forms of Arnold [1, §8.4] gives another and very appealing view of centre manifold reduction. Corresponding to normal forms for deterministic systems, a normal form of stochastic dynamics empowers us simultaneously to extract the SCM, derive the stochastic evolution thereon, and show asymptotic completeness. However, I believe that further research will simplify the memory convolutions (as seen in Appendix B) which have so far appeared in normal forms of stochastic dynamics.

\section{Appendix B. Quadratic stochastic effects: alternative derivation}

This Appendix uses the Ito interpretation of SDEs, rather than the Stratonovich interpretation used throughout the body of this work. We consider some of the properties of quadratic noise interactions that in Sections 4 and 5 were established through analysis of Fokker-Planck equations. Here we provide alternative, more direct, derivations of the effective drift, volatility and inter-dependencies of the long-time dynamics of the $\vec{y}$ variables in the canonical interaction system of SDEs (25).

\section{B.1. Noise interacting with itself over long times}

As a prelude to more extensive analysis, this subsection begins by analysing the simplest case of one noise process quadratically interacting with itself; that is, $\phi_{i}=\phi_{j}$. Thus we explore the large-time dynamics of the first pair of Stratonovich SDEs in the system (25). The equivalent Ito SDEs, written in the more usual capital letters, are for some Wiener process $W$

$$
d Y=\frac{1}{2} d t+Z d W \quad \text { and } \quad d Z=-\beta Z d t+d W,
$$

where all subscripts are omitted for simplicity, $Y=y_{1}, Z=z_{1}$ and $d W=\phi_{j} d t=\phi_{i} d t$. 
Consider the dynamics over any time interval $[a, b]$. Where necessary, the times $a$ and $b$ are large enough for exponentially decaying transients to become negligible. For notational simplicity, we use just $\int$ to denote $\int_{a}^{b}$ and just $\Delta$ to denote the difference []$_{t=a}^{t=b}$.

Proposition 6. The process $Y$ has drift $\frac{1}{2}$ with a variance growing linearly at a rate $1 /(2 \beta)$.

An argument to support this proposition is the following. Integrate the $Y$ sDE to $\Delta Y=$ $\frac{1}{2} \Delta t+\int Z d W$ and take expectations:

$$
\mathrm{E}[\Delta Y]=\frac{1}{2} \Delta t+\mathrm{E}\left[\int Z d W\right]=\frac{1}{2} \Delta t,
$$

by the martingale property of Ito integrals. Hence $Y$ has drift $\frac{1}{2}$.

Now consider

$$
\begin{aligned}
\operatorname{Var}\left[\Delta\left(Y-\frac{1}{2} t\right)\right] & =\operatorname{Var}\left[\int Z d W\right] \\
& =\int \mathrm{E}\left[Z^{2}\right] d t \quad \text { by Ito isometry } \\
& =\frac{\Delta t}{2 \beta},
\end{aligned}
$$

as $Z$ is a well known Ornstein-Uhlenbeck process. Hence the variance of $Y$ grows linearly at rate $1 /(2 \beta)$.

Rather than appeal to $Z$ being an Ornstein-Uhlenbeck process, we could instead recognise that

$$
Z=\int_{-\infty}^{t} \exp \{-\beta(t-s)\} d W_{S}
$$

from the defining convolution; then

$$
\mathrm{E}\left[Z^{2}\right]=\operatorname{Var}\left[\int_{-\infty}^{t} \exp \{-\beta(t-s)\} d W_{s}\right]
$$

which by the Ito isometry is equal to

$$
\begin{aligned}
& \int_{-\infty}^{t} \mathrm{E}\left[\exp \{-\beta(t-s)\}^{2}\right] d s \\
= & \int_{-\infty}^{t} \exp \{-2 \beta(t-s)\} d s \\
= & \frac{1}{2 \beta},
\end{aligned}
$$

which confirms the growth of the variance. The next subsection uses this route to find covariances with any number of convolutions.

Given that $\Delta Y$ approaches a Gaussian over long timescales, as established in the FokkerPlanck analysis leading to (17)-(18) and shown in some numerical simulations by Chao \& Roberts [10], the process $Y$ may be thus modelled over long timescales by the SDE

$$
d Y=\frac{1}{2} d t+\frac{1}{\sqrt{2 \beta}} d W_{1}
$$

for some Wiener process $W_{1}$, as analogously derived in (19). 
However, before this corollary is of any use we need to establish that the Wiener process $W_{1}$ is effectively independent of the original Weiner process $W$ when viewed over large timescales. The next proposition asserts the correlation $\mathrm{E}\left[\Delta W \cdot \Delta W_{1}\right]=0$.

Proposition 7. For the processes $Y$ and $Z$ with Ito SDE (29), the correlation

$$
\mathrm{E}\left[\Delta W \cdot \Delta\left(Y-\frac{1}{2} t\right)\right]=0,
$$

and hence the increments $\Delta W$ and $\Delta\left(Y-\frac{1}{2} t\right)$ are independent.

An argument to support this proposition is the following. Since trivially $\mathrm{E}[\Delta W \cdot \Delta t]=$ 0 , we need only consider $\mathrm{E}[\Delta W \cdot \Delta Y]$. Since $\left(W-W_{a}\right)\left(Y-Y_{a}\right)=0$ at $t=a$, it follows that

$$
\Delta W \cdot \Delta Y=\Delta\left\{\left(W-W_{a}\right)\left(Y-Y_{a}\right)\right\}
$$

Hence

$$
\begin{aligned}
\mathrm{E}[\Delta W \cdot \Delta Y] & =\mathrm{E}\left[\Delta\left\{\left(W-W_{a}\right)\left(Y-Y_{a}\right)\right\}\right] \\
& =\mathrm{E}\left[\int d\left\{\left(W-W_{a}\right)\left(Y-Y_{a}\right)\right\}\right]
\end{aligned}
$$

which by Ito's formula (see, for example, [3, p. 62]) is equal to

$$
\begin{aligned}
& \mathrm{E}\left[\int Y-Y_{a}+Z\left(W-W_{a}\right) d W+\int Z+\frac{1}{2}\left(W-W_{a}\right) d t\right] \\
= & \mathrm{E}\left[\int Y-Y_{a}+Z\left(W-W_{a}\right) d W\right]+\int \mathrm{E}[Z]+\frac{1}{2} \mathrm{E}\left[W-W_{a}\right] d t \\
= & 0,
\end{aligned}
$$

by the martingale property of Ito integrals, by the fact that $Z$ is an Ornstein-Uhlenbeck process and hence has zero expectation except for exponentially decaying transients, and since Wiener increments have zero expectation. Consequently, the increments $\Delta W$ and $\Delta\left(Y-\frac{1}{2} t\right)$ are independent.

\section{B.2. Two distinct and interacting noises}

Now turn to the case of two noise processes interacting; that is, when $\phi_{i} \neq \phi_{j}$. Thus we explore the large-time dynamics of the first pair of Stratonovich SDEs in the system (25). Now the equivalent Ito SDEs for some independent Wiener processes $W$ and $\hat{W}$ are

$$
d Y=Z d W \quad \text { and } \quad d Z=-\beta Z d t+d \hat{W},
$$

where $d W=\phi_{j} d t$ and $d \hat{W}=\phi_{i} d t$.

As in the argument for Proposition 6, we write the increments $\Delta Y=\int Z d W$, and then the martingale property and the Ito isometry assure us that $\mathrm{E}[\Delta Y]=0$ and $\operatorname{Var}[\Delta Y]=$ $\Delta t /(2 \beta)$. Similar to the argument for Proposition 7, the increment $\Delta Y$ is uncorrelated with both $\Delta W$ and $\Delta \hat{W}$; we use Ito's formula for products of processes that depend upon multiple 
noises (see, for example, [3, p.185]):

$$
\begin{aligned}
\mathrm{E}[\Delta W \cdot \Delta Y] & =\mathrm{E}\left[\int d\left\{\left(W-W_{a}\right)\left(Y-Y_{a}\right)\right\}\right] \\
& =\mathrm{E}\left[\int\left(W-W_{a}\right) Z+\left(Y-Y_{a}\right) d W+\int Z d t\right] \\
& =\mathrm{E}\left[\int\left(W-W_{a}\right) Z+\left(Y-Y_{a}\right) d W\right]+\int \mathrm{E}[Z] d t \\
& =0 ; \\
\mathrm{E}[\Delta \hat{W} \cdot \Delta Y] & =\mathrm{E}\left[\int d\left\{\left(\hat{W}-\hat{W}_{a}\right)\left(Y-Y_{a}\right)\right\}\right] \\
& =\mathrm{E}\left[\int\left(\hat{W}-\hat{W}_{a}\right) Z d W+\int Y-Y_{a} d \hat{W}\right] \\
& =\mathrm{E}\left[\int\left(W-W_{a}\right) Z d W\right]+\mathrm{E}\left[\int Y-Y_{a} d \hat{W}\right] \\
& =0 .
\end{aligned}
$$

Consequently, given that $\Delta Y$ approaches a Gaussian over long timescales, we may model the process $Y$ by the SDE $d Y=(1 / \sqrt{2 \beta}) d W_{1}$ for some effectively independent Wiener process $W_{1}$, as analogously derived in (19).

This subsection gives alternative and more direct proofs of some of the Fokker-Planck analysis of Section 4 on the most elementary canonical noise interactions. However, this subsection does not establish the key property that the increments $\Delta Y$ approach a Gaussian for long times. Instead, the relevance theorem of centre manifolds, together with the structural stability of the Fokker-Planck equation (17), assures us of this key property.

\section{B.3. Multiple convolutions of quadratic noises}

To complete the analysis, we here explore noise processes interacting with multiple convolutions of their past history. Thus we consider the Ito version of the Stratonovich hierarchy of SDEs (25):

$$
\begin{aligned}
d Y_{1} & =\frac{1}{2} s d t+Z_{1} d W, & d Z_{1} & =-\beta_{1} Z_{1} d t+d \hat{W}, \\
d Y_{2} & =Z_{2} d W, & d Z_{2} & =\left(-\beta_{2} Z_{2}+Z_{1}\right) d t, \\
& \vdots & & \vdots \\
d Y_{n} & =Z_{n} d W, & d Z_{n} & =\left(-\beta_{n} Z_{n}+Z_{n-1}\right) d t,
\end{aligned}
$$

where $W=\hat{W}$ in the case of a noise interacting with itself, $s=1$; otherwise they are independent, $s=0$. Ito calculus provides an alternative confirmation to that derived in Section 5, of the effective large-time dynamics of the processes $Y_{m}$.

The processes $Y_{m}$ have zero drift except for the case $W=\hat{W}$ when instead process $Y_{1}$ has drift $\frac{1}{2}$. We need the covariances of the fluctuations in these processes in order to establish that the correlations among the fluctuations is determined by the lower triangular matrix $\mathbb{L}$ in (28). For conciseness, we define the fluctuation process $\mathfrak{Y}_{m}=Y_{m}-\delta_{m 1} \frac{1}{2} s t$.

Proposition 8. The expectation $\mathrm{E}\left[\Delta \mathfrak{Y}_{m}\right]=0$ for all $m$, and the covariances $\mathrm{E}\left[\Delta \mathfrak{Y}_{k} \Delta \mathfrak{Y}_{m}\right]$ are the corresponding elements in $\Delta t \mathbb{L L}^{T}$ for the lower triangular matrix $\mathbb{L}$ in (28). 
An argument to support this proposition is the following. Firstly, immediately from the definition of $\mathfrak{Y}_{m}$ and the Ito SDEs (31), we have $d \mathfrak{Y}_{m}=Z_{m} d W$. Recall that an unadorned $\int$ denotes $\int_{a}^{b}$ and $\Delta$ denotes the difference []$_{t=a}^{t=b}$. Thus $\Delta \mathfrak{Y}_{m}=\int Z_{m} d W$, and then $\mathrm{E}\left[\Delta \mathfrak{Y}_{m}\right]=0$ by the martingale property of an Ito integral.

Secondly, consider the covariances

$$
\mathrm{E}\left[\Delta \mathfrak{Y}_{k} \Delta \mathfrak{Y}_{m}\right]=\mathrm{E}\left[\int Z_{k} d W \int Z_{m} d W\right]=\int \mathrm{E}\left[Z_{k} Z_{m}\right] d t
$$

by an extension of the Ito isometry.

Find these covariances by observing (and this is actually the definition from convolutions of the right-hand column in the hierarchy of Ito SDEs (31)) that

$$
Z_{1}=\int_{-\infty}^{t} e^{-\beta_{1}(t-s)} d \hat{W}_{s} \quad \text { and } \quad Z_{m}=\int_{-\infty}^{t} e^{-\beta_{m}(t-s)} Z_{m-1}(s) d s .
$$

The first is an Ito integral. Turn the others into Ito integrals by defining

$$
h_{1}(t)=e^{-\beta_{1} t} \quad \text { and } \quad h_{m}(t)=e^{-\beta_{m} t} \star h_{m-1}(t)=\int_{0}^{t} e^{-\beta_{m}(t-s)} h_{m-1}(s) d s ;
$$

for example, when the decay rates $\beta_{m}$ are distinct, then

$$
\begin{aligned}
& h_{2}(t)=\frac{e^{-\beta_{2} t}-e^{-\beta_{1} t}}{\beta_{1}-\beta_{2}}, \\
& h_{3}(t)=\frac{e^{-\beta_{1} t}}{\left(\beta_{1}-\beta_{2}\right)\left(\beta_{1}-\beta_{3}\right)}+\frac{e^{-\beta_{2} t}}{\left(\beta_{2}-\beta_{3}\right)\left(\beta_{2}-\beta_{1}\right)}+\frac{e^{-\beta_{3} t}}{\left(\beta_{3}-\beta_{1}\right)\left(\beta_{3}-\beta_{2}\right)} .
\end{aligned}
$$

Then inductively

$$
\begin{aligned}
Z_{m} & =\int_{-\infty}^{t} e^{-\beta_{m}(t-\tau)} \int_{-\infty}^{\tau} h_{m-1}(\tau-s) d \hat{W}_{s} d \tau \\
& =\int_{-\infty}^{t} \int_{s}^{t} e^{-\beta_{m}(t-\tau)} h_{m-1}(\tau-s) d \tau d \hat{W}_{s} \\
& =\int_{-\infty}^{t} \int_{0}^{t-s} e^{-\beta_{m}(t-s-\tau)} h_{m-1}(\tau) d \tau d \hat{W}_{s} \\
& =\int_{-\infty}^{t} h_{m}(t-s) d \hat{W}_{s} .
\end{aligned}
$$

Consequently, by an extension of the Ito isometry, we have

$$
\begin{aligned}
\mathrm{E}\left[Z_{m} Z_{k}\right] & =\mathrm{E}\left[\int_{-\infty}^{t} h_{m}(t-s) d \hat{W}_{s} \int_{-\infty}^{t} h_{k}(t-s) d \hat{W}_{s}\right] \\
& =\int_{-\infty}^{t} \mathrm{E}\left[h_{m}(t-s) h_{k}(t-s)\right] d s \\
& =\int_{0}^{\infty} h_{m}(t) h_{k}(t) d t .
\end{aligned}
$$

Computer algebra [36, §3] readily computes the convolutions and integrals in equations (33) and (34). The resultant covariances $\mathrm{E}\left[Z_{k} Z_{m}\right]$ are correctly twice the corresponding elements in the diffusion matrices $\mathbb{D}$ given in (18) and (27). 
The computer algebra [36, §3] easily computes higher-order covariance matrices, but the expressions for order $n \geqslant 4$ are too hideous to record in detail here. However, (28) records the expressions computed for the fourth-order Cholesky factorisation.

The Cholesky factorisation (28) is needed to weakly model convolutions of noise by effectively new and independent noises, as discussed in Section 4. Again, we need to be sure that these effectively new noise processes are independent of the original processes $W$ and $\hat{W}$.

Proposition 9. For the processes $Y_{m}$ and $Z_{m}$ with Ito SDE (31), the correlation

$$
\left.\left.\mathrm{E}\left[\Delta W \cdot \Delta \mathfrak{Y}_{m}\right)\right]=\mathrm{E}\left[\Delta \hat{W} \cdot \Delta \mathfrak{Y}_{m}\right)\right]=0,
$$

and hence the increments $\Delta W, \Delta \hat{W}$ and $\Delta \mathfrak{Y}_{m}$ are independent.

An argument to support this proposition is the following. As in Proposition 7, since $\left(W-W_{a}\right)\left(\mathfrak{Y}_{m}-\mathfrak{Y}_{m a}\right)=0$ at $t=a$, it follows that

$$
\Delta W \cdot \Delta \mathfrak{Y}_{m a}=\Delta\left\{\left(W-W_{a}\right)\left(\mathfrak{Y}_{m}-\mathfrak{Y}_{m a}\right)\right\} .
$$

Hence, using Ito's formula for products of processes that depend upon multiple noises (see, for example, [3, p. 185]), we have

$$
\begin{aligned}
\mathrm{E}\left[\Delta W \cdot \Delta \mathfrak{Y}_{m}\right] & =\mathrm{E}\left[\Delta\left\{\left(W-W_{a}\right)\left(\mathfrak{Y}_{m}-\mathfrak{Y}_{m a}\right)\right\}\right] \\
& =\mathrm{E}\left[\int d\left\{\left(W-W_{a}\right)\left(\mathfrak{Y}_{m}-\mathfrak{Y}_{m a}\right)\right\}\right] \\
& =\mathrm{E}\left[\int \mathfrak{Y}_{m}-\mathfrak{Y}_{m a}+Z_{m}\left(W-W_{a}\right) d W+\int Z_{m} d t\right] \\
& =\mathrm{E}\left[\int \mathfrak{Y}-\mathfrak{Y}_{m a}+Z_{m}\left(W-W_{a}\right) d W\right]+\int \mathrm{E}\left[Z_{m}\right] d t \\
& =0,
\end{aligned}
$$

by the martingale property of Ito integrals, including $Z_{m}=\int_{-\infty}^{t} h_{m}(t-s) d \hat{W}_{s}$ as deduced above. Consequently, the increments $\Delta W$ and $\Delta \mathfrak{Y}_{m}$ are independent.

Similarly,

$$
\begin{aligned}
\mathrm{E}\left[\Delta \hat{W} \cdot \Delta \mathfrak{Y}_{m}\right] & =\mathrm{E}\left[\Delta\left\{\left(\hat{W}-\hat{W}_{a}\right)\left(\mathfrak{Y}_{m}-\mathfrak{Y}_{m a}\right)\right\}\right] \\
& =\mathrm{E}\left[\int d\left\{\left(\hat{W}-\hat{W}_{a}\right)\left(\mathfrak{Y}_{m}-\mathfrak{Y}_{m a}\right)\right\}\right] \\
& =\mathrm{E}\left[\int \mathfrak{Y}_{m}-\mathfrak{Y}_{m a} d \hat{W}+\int\left(\hat{W}-\hat{W}_{a}\right) Z_{m} d W\right] \\
& =\mathrm{E}\left[\int \mathfrak{Y}-\mathfrak{Y}_{m a} d \hat{W}\right]+\mathrm{E}\left[\int\left(\hat{W}-\hat{W}_{a}\right) Z_{m} d W\right] \\
& =0,
\end{aligned}
$$

by the martingale property of Ito integrals. Consequently, the increments $\Delta \hat{W}$ and $\Delta \mathfrak{Y}_{m}$ are independent. 


\section{References}

1. Ludwig Arnold, Random dynamical systems, Springer Monogr. Math. (Springer, 2003) ISBN 3-540-63758-3. 194, 195, 196, 211, 213

2. L. Arnold, N. Sri Namachchivaya and K. R. Schenk-Hoppé, 'Toward an understanding of stochastic Hopf bifurcation: a case study', Internat. J. Bifur. Chaos Appl. Sci. Engrg. 6 (1996) 1947-1975. 197

3. Martin BAXter and Andrew Rennie, Financial calculus: An introduction to derivative pricing (Cambridge University Press, 1996). 215, 216, 218

4. A. Bensoussan and F. Flandoli, 'Stochastic inertial manifold', Stochastics Stochastics Rep. 53 (1995) 13-39. 195

5. Nils Berglund and Barbara Gentz, 'Geometric singular perturbation theory for stochastic differential equations', J. Differential Equations 191 (2003) 1-54, http://dx.doi.org/10.1016/s0022-0396(03)00020-2. 194

6. D. Blomker, M. Hairer and G. A. Pavliotis, 'Modulation equations: stochastic bifurcation in large domains', Comm. Math. Phys. 258 (2005) 479-512, http://dx.doi.org/10.1007/s00220-005-1368-8.193, 194, 195

7. P. BoXler, 'A stochastic version of the centre manifold theorem', Probab. Theory Related Fields 83 (1989) 509-545. 193, 194, 195, 196, 199, 211, 212, 213

8. P. BoXler, How to construct stochastic center manifolds on the level of vector fields, Lecture Notes in Math. 1486 (Springer, 1991) 141-158. 194

9. Tomas Caraballo, Jose A. Langa and James C. Robinson, 'A stochastic pitchfork bifurcation in a reaction-diffusion equation', Proc. Roy. Soc. Lond. A 457 (2001) 2041-2061, http://dx.doi.org/10.1098/rspa.2001.0819.195

10. Xu Chao and A. J. Roberts, 'On the low-dimensional modelling of Stratonovich stochastic differential equations', Physica A 225 (1996) 62-80,

http://dx.doi.org/10.1016/0378-4371(95)00387-8. 197, 198, 200, 201, 202, 204, 205, 214

11. P. C. Chatwin, 'The approach to normality of the concentration distribution of a solute in a solvent flowing along a straight pipe', J. Fluid Mech. 43 (1970) 321-352. 205

12. C. Chicone and Y. Latushin, 'Center manifolds for infinite dimensional nonautonomous differential equations', J. Differential Equations 141 (1997) 356-399,

http://www.ingentaconnect.com/content/ap/de/1997/00000141/ $00000002 / \operatorname{art} 03343.200$

13. P. H. Coullet, C. Elphick and E. Tirapegui, 'Normal form of a Hopf bifurcation with noise', Phys. Lett. 111A (1985) 277-282. 197, 198

14. Francois Drolet and Jorge Vinals, 'Adiabatic reduction near a bifurcation in stochastically modulated systems', Phys. Rev. E 57 (1998) 5036-5043, http://link.aps.org/abstract/PRE/v57/p5036. 196, 201, 202

15. Francois Drolet and Jorge Vinals, 'Adiabatic elimination and reduced probability distribution functions in spatially extended systems with a fluctuating control parameter', Phys. Rev. E 64 (2001) 026120, http://link.aps.org/abstract/PRE/v64/e026120. 193, 196

16. Jinqiao Duan, Kening Lu and BJorn Schmalfuss, 'Invariant manifolds for stochastic partial differential equations', Ann. Probab. 31 (2003) 2109-2135. 195 
17. Th. Gallay, 'A center-stable manifold theorem for differential equations in Banach spaces', Comm. Math. Phys 152 (1993) 249-268. 202

18. W. Grecksch and P. E. Kloeden, 'Time-discretised Galerkin approximations of parabolic stochastics PDEs', Bull. Austral. Math. Soc. 54 (1996) 79-85. 193

19. Wolfram Just, Holger Kantz, Christian Rodenbeck and Mario Helm, 'Stochastic modelling: replacing fast degrees of freedom by noise', J. Phys. A: Math. Gen. 34 (2001) 3199-3213.196, 197, 201, 202, 203, 204, 207, 210

20. Yuri Kabanov and Sergei Pergamenshchikov, Two-scale stochastic systems, Applications of Mathematics: Stochastic Modelling and Applied Probability 49 (Springer, 2003). 194

21. P. E. Kloeden and E. Platen, Numerical solution of stochastic differential equations, Appl. Math. 23 (Springer, 1992). 193

22. E. KNOBLOCH and K. A. WiESENFELD, 'Bifurcations in fluctuating systems: The centre manifold approach', J. Statist. Phys 33 (1983) 611-637.193, 194, 196

23. T. MackenzIE and A. J. Roberts, 'Holistic finite differences accurately model the dynamics of the Kuramoto-Sivashinsky equation', ANZIAM J. 42 (2000) C918-C935, http://anziamj.austms.org.au/V42/CTAC99/Mack. 194

24. T. MacKenzie and A. J. Roberts, 'Holistic discretisation of shear dispersion in a two-dimensional channel', Proc., 10th Computational Techniques and Applications Conference CTAC-2001, ed. K. Burrage and Roger B. Sidje, ANZIAM J. 44C (2003) C512-C530, http: //anziamj.austms.org.au/V4 4/CTAC2001/Mack.194, 211

25. R. Metzler, 'Non-homogeneous random walks, generalised master equations, fractional Fokker-Planck equations, and the generalised Kramers-Moyal expansion', Eur. Phys. J. B 19 (2001) 249-258,

http://www.edpsciences.org/articles/epjb/pdf/2001/02/b0331.pdf. 203

26. A. NAert, R. Friedrich and J. Peinke, 'Fokker-Planck equation for the energy cascade in turbulence', Phys. Rev. E 56 (1997) 6719-6722.203

27. P. K. Pollett and A. J. Roberts, 'A description of the long-term behaviour of absorbing continuous time Markov chains using a centre manifold', Adv. in Appl. Probab. 22 (1990) 111-128.197, 202

28. A. J. Roberts, 'The application of centre manifold theory to the evolution of systems which vary slowly in space', J. Austral. Math. Soc. B 29 (1988) 480-500.202, 203

29. A. J. Roberts, 'Low-dimensional modelling of dynamics via computer algebra', Comput. Phys. Comm. 100 (1997) 215-230. 197, 198, 211

30. A. J. Roberts, 'Holistic discretisation ensures fidelity to Burgers' equation', Appl. Numer. Modelling 37 (2001) 371-396, http://arXiv.org/abs/chao-dyn/9901011.194, 211

31. A. J. Roberts, 'Holistic projection of initial conditions onto a finite difference approximation', Comput. Phys. Comm. 142 (2001) 316-321, http://arXiv.org/abs/math. NA/0101205.194

32. A. J. Roberts, 'A holistic finite difference approach models linear dynamics consistently’, Math. Comp. 72 (2002) 247-262, http://arXiv.org/abs/math.NA/0003135. 194, 211 
33. A. J. Roberts, 'Derive boundary conditions for holistic discretisations of Burgers' equation', Proc., 10th Computational Techniques and Applications Conference CTAC-2001, ed. K. Burrage and Roger B. Sidje, ANZIAM J. 44C (2003) C664-C686, http://anziamj.austms.org.au/V44/CTAC2001/Robe. 194

34. A. J. Roberts, 'Low-dimensional modelling of dynamical systems applied to some dissipative fluid mechanics', Nonlinear dynamics from lasers to butterflies, ed. Rowena Ball and Nail Akhmediev, Lecture Notes in Complex Systems 1 (World Scientific, 2003) 257-313. 202, 203

35. A. J. RoberTs, 'A step towards holistic discretisation of stochastic partial differential equations', Proc., 11th Computational Techniques and Applications Conference CTAC-2003 (Dec. 2003), ed. Jagoda Crawford and A. J. Roberts, ANZIAM J. 45 (2004) C1-C15, http: / / anziamj.austms.org.au/V45/CTAC2003/Robe. 193, 194, 196, 197, 198, 199, 200, 201, 202, 204, 207

36. A. J. Roberts, 'Computer algebra resolves a multitude of microscale interactions to model stochastic partial differential equations', Technical report, University of Southern Queensland, December 2005,

http://www.sci.usq.edu.au/staff/robertsa/CA/multinoise.pdf. 197, 200, 203, 206, 208, 209, 217, 218

37. J. C. Robinson, 'The asymptotic completeness of inertial manifolds', Nonlinearity 9 (1996) 1325-1340, http: / / www. iop.org/EJ/abstract/0 951-7715/9/5/013. 212

38. G. SchÖNER and H. HAKEN, 'The slaving principle for Stratonovich stochastic differential equations', Z. Phys. B - Condensed matter 63 (1986) 493-504. 197, 202

39. N. Sri Namachchivaya and Y. K. Lin, 'Method of stochastic normal forms', Int. J. Nonlinear Mechanics 26 (1991) 931-943. 197, 198

40. M. TutKUn and L. Mydlarski, 'Markovian properties of passive scalar increments in grid-generated turbulence', New J. Phys. 6 (2004) article 49, 1-24, http://dx.doi.org/10.1088/1367-2360/6/1/049. 203

41. ERIC VANDEn-EIJnden, 'Asymptotic techniques for SDEs', Fast times and fine scales: Proc., 2005 Program in Geophysical Fluid Dynamics (Woods Hole Oceanographic Institution, 2005),

http://gfd.whoi.edu/proceedings/2005/PDFvol2005.html. 193, 196, 202, 207

42. M. J. Werner and P. D. Drummond, 'Robust algorithms for solving stochastic partial differential equations', J. Comput. Phys. 132 (1997) 312-326. 193

A. J. Roberts aroberts@usq.edu.au

http://www.sci.usq.edu.au/staff/aroberts

Research Centre

Department of Mathematics \& Computing

University of Southern Queensland

Toowoomba

Queensland 4352

Australia. 